\title{
Magnetization of the intergalactic medium in the IllustrisTNG simulations: the importance of extended, outflow-driven bubbles
}

\author{
Andrés Arámburo-García ${ }^{1 \star}$, Kyrylo Bondarenko ${ }^{2,3} \dagger$, Alexey Boyarsky $^{1} \ddagger$, \\ Dylan Nelson ${ }^{4} \S$, Annalisa Pillepich ${ }^{5}$, and Anastasia Sokolenko ${ }^{6} \|$ \\ ${ }^{1}$ Institute Lorentz, Leiden University, Niels Bohrweg 2, Leiden, NL-2333 CA, the Netherlands \\ ${ }^{2}$ Theoretical Physics Department, CERN, Geneva 23, CH-1211, Switzerland \\ ${ }^{3}$ L'Ecole polytechnique fédérale de Lausanne, 1015 Lausanne, Switzerland \\ ${ }^{4}$ Universität Heidelberg, Zentrum für Astronomie, Institut für theoretische Astrophysik, Albert-Ueberle-Str. 2, 69120 Heidelberg, Germany \\ 5 Max-Planck-Institut für Astronomie, Königstuhl 17, 69117 Heidelberg, Germany \\ ${ }^{6}$ Institute of High Energy Physics, Austrian Academy of Sciences, Nikolsdorfergasse 18, 1050 Vienna, Austria
}

June 30, 2021

\begin{abstract}
We study the effects of galaxy formation physics on the magnetization of the intergalactic medium (IGM) using the IllustrisTNG simulations. We demonstrate that large-scale regions affected by the outflows from galaxies and clusters contain magnetic fields that are several orders of magnitude stronger than in unaffected regions with the same electron density. Moreover, like magnetic fields amplified inside galaxies, these magnetic fields do not depend on the primordial seed, i.e. the adopted initial conditions for magnetic field strength. We study the volume filling fraction of these strong field regions and their occurrence in random lines of sight. As a first application, we use these results to put bounds on the photon-axion conversion from spectral distortion of the CMB. As photon-axion coupling grows with energy, stronger constraints could potentially be obtained using data on the propagation of gamma-ray photons through the IGM. Finally, we also briefly discuss potential applications of our results to the Faraday Rotation measurements.
\end{abstract}

Key words: magnetic fields, intergalactic medium, MHD, large-scale structure of the Universe, simulations, astroparticle physics

\section{INTRODUCTION}

The Universe is magnetized on all scales - from planets and stars to galaxy clusters and beyond. Magnetic fields affect the propagation of charged particles and therefore play an important role in the physics of the Earth's atmosphere, the Sun and solar system, galaxies, and so on. However, the origin of magnetic fields in galaxies and, especially, galaxy clusters in the low-redshift Universe remains an important open problem (see e.g. Durrer \& Neronov 2013 for a review).

Indeed, there are two stages of magnetogenesis - the first stage is the generation of weak seed magnetic fields and the second stage is their subsequent evolution during structure formation. Seed fields could have either primordial (produced before recombination) or astrophysical origin (produced after the formation of the first stars), see e.g. Subramanian (2016) for a review. Primordial seed fields fill

\footnotetext{
*aramburo@strw.leidenuniv.nl

† kyrylo.bondarenko@cern.ch

† boyarsky@lorentz.leidenuniv.nl

$\S$ dnelson@uni-heidelberg.de

ๆ pillepich@mpia-hd.mpg.de

\| anastasia.sokolenko@oeaw.ac.at
}

the whole universe, although they are not necessarily constant, their correlation length depends on the production mechanism and the epoch when they were produced. An astrophysical seed magnetic field is generated by Biermann battery-type mechanisms when the curl of the electric field is created by non-parallel gradients of density and temperature, giving rise to a magnetic field via Faraday induction (Subramanian 2016). Such a mechanism could generate magnetic fields during star formation, reionization (Subramanian et al. 1994; Gnedin et al. 2000), or even during the later collapse of galaxies and halos in cosmological shocks (Kulsrud et al. 1997). For example, Vazza et al. (2017) simulate 25 different scenarios of initial magnetogenesis, both primordial and astrophysical.

In the second stage, magnetic fields evolve with the expansion of the Universe and structure formation. Outside structures, these fields dilute approximately as $\sim a^{-2}$ (Durrer \& Neronov 2013), where $a$ is the scale factor. In the regions where dense structures form, magnetic fields are adiabatically compressed and, moreover, strongly amplified (up to several $\mu \mathrm{G}$ Pakmor et al. 2014a; Rieder \& Teyssier 2017) by different dynamo mechanisms driven by the baryonic physics of galaxy formation (Parker 1955; Ruzmaikin et al. 1988; Kulsrud 1999; Brandenburg \& Subramanian 2005; Kulsrud \& Zweibel 2008; Rieder \& Teyssier 2016; Su et al. 
2017; Butsky et al. 2017; Steinwandel et al. 2019; Martin-Alvarez et al. 2018; Vazza et al. 2018). Amplification in filaments also occurs via shear flows (Birk et al. 1999; Dolag et al. 1999; Dolag et al. 2005). As a result, magnetic fields in galaxies and collapsed structures, amplified by many orders of magnitude by gravomagnetohydrodynamics (MHD) dynamos, "forget" the properties of the initial magnetic fields (see e.g. Pakmor et al. 2014a; Marinacci et al. 2015; Pillepich et al. 2018a, for cosmological simulations of galaxies). To the contrary, magnetic fields that are far from structures are much closer to the diluted initial fields and could therefore be used to infer information about the properties and the origin of the initial fields.

On the observational side, cosmic magnetic fields are relatively well studied in virialized objects like galaxies and galaxy clusters. A powerful method to detect these fields is Faraday Rotation Measure (RM) see e.g. Brentjens \& de Bruyn (2005) and references therein. With the current generation of instruments, this method is efficient for magnetic fields with the strength of $B \geqslant \mathcal{O}(1) \mathrm{nG}$ (Durrer \& Neronov 2013). Such magnetic fields exist mainly in the dense centers of collapsed structures - galaxies, galaxy groups, and clusters (e.g. Carilli \& Taylor 2002; Laing et al. 2008; Beck 2015; van Weeren et al. 2019). However, these objects fill only a small fraction of the volume of the Universe.

Empirical constraints on the cosmic magnetic fields outside galaxies and clusters remain difficult. Attempts to measure magnetic fields in filaments by Faraday Rotation Measure with LOFAR (O'Sullivan et al. 2020) place only an upper bound of a few nG, on Mpc scales, consistent with other works (Ravi et al. 2016; Vernstrom et al. 2019; Blasi et al. 1999; Pshirkov et al. 2016; Hackstein et al. 2016; Bray \& Scaife 2018). In addition, a lower bound can be obtained with high-energy gamma-ray data (Neronov \& Vovk 2010; Dermer et al. 2011; Tavecchio et al. 2011; Dolag et al. 2011; Taylor et al. 2011). This lower bound is as weak as $B \gtrsim 10^{-17} \mathrm{G}$ on Mpc scales. In the future, the upper bound can be improved with next-generation radio telescopes such as the Square Kilometer Array (SKA) (Carilli \& Rawlings 2004), while the lower bound may be improved by high-energy observatories including CTA (Acharya et al. 2018), which is expected to start obtaining data soon. For now, the observational uncertainty in the properties of the intergalactic magnetic fields (IGMF) outside galactic halos is large.

From the theoretical as well as observational perspective our knowledge on the magnetic fields outside galaxies and clusters is rather limited. At the same time, magnetic fields in the large volumes of the intergalactic medium (IGM) can play a profound role in many important problems in physics. For example, magnetic fields can strongly affect the propagation of light and the spectra of various astrophysical sources (see e.g. Brentjens \& de Bruyn 2005; Neronov \& Vovk 2010) as well as propagation of cosmic rays (Alves Batista et al. 2017). If the magnetic field along the lines of sight to certain classes of sources were better constrained, these effects (see examples below) can give us important insight into fundamental physics.

When light propagates towards us from remote sources, most of the intervening pathlength is typically not in virialized objects, as these occupy only a tiny fraction of the Universe by volume. Rather, photons propagate through the IGM - the space between dark matter halos occupied by less dense regions including cosmic voids, sheets, and filamentary structures. Both the free electron number density and magnetic field strength in the IGM evolve with time, and theoretical modeling of this evolution, through the epoch of reionization and down to the present day, remains a challenge.
Therefore, to probe the effects of cosmic magnetic fields on light propagation it is not enough to model magnetic fields only within halos.

One regime where magnetic fields in the IGM play a crucial role is gamma-ray astronomy. The photons from high-energy gamma-ray sources create electron-positron pairs interacting with the extra-galactic background light. These charged particles can then emit secondary gamma-ray photons interacting with the CMB via the inverse Compton effect. The presence of a magnetic field results in a deviation of the charged particles and, therefore, in a change in the morphology of the signal (see e.g. Neronov \& Semikoz 2009 for a more detailed discussion). ${ }^{1}$

Magnetic fields can also affect light propagation in the presence of new, as of yet unobserved particles that are not included in the Standard Model of particle physics. A famous example is an axion or axion-like particle (ALP), initially introduced to explain why CP violation in QCD is so tiny (Weinberg 1978; Wilczek 1978). Axions have been theorized to play the role of dark matter (Preskill et al. 1983). Photons can be converted into ALPs, but only when they pass through magnetized regions of the Universe. In this case, the conversion probability depends sensitively on the strength of the magnetic field (Sikivie 1983; Raffelt \& Stodolsky 1988).

In this paper, we use the IllustrisTNG suite of cosmological simulations (see Section 2 for details), as well as additional variation runs performed with different values of the initial magnetic fields and with and without feedback, to study the regions of the IGM that could be affected by galactic outflows. The starting point of our investigation is the idea that the strong magnetic fields generated deep within dark matter halos can affect and extend to much larger volumes, as baryonic outflows, caused by strong feedback processes, eject magnetized gas to regions extending far beyond halo scales (and not just beyond galactic scales, as studied e.g. in Marinacci et al. 2018; Steinwandel et al. 2020; Pakmor et al. 2020; Dubois \& Teyssier 2010; Martin-Alvarez et al. 2020).

In this work, we concentrate our attention on the regions of the IGM affected by galactic outflows, their origin, and their impact on magnetic fields in the IGM. We discuss the properties and strength of the IGMF predicted in IllustrisTNG (Section 2), presenting our results in a form that may be used for different applications. We show that the magnetic fields affected by galactic outflows depend more on the MHD processes occurring within galaxies rather than on the primordial magnetic field seeds. In particular, we show that the predicted magnetic field strength in the regions affected by outflows is similar for runs spanning orders of magnitude different strength of the initial magnetic fields (Section 3). At the same time, magnetic fields in these regions are orders of magnitude larger than in other regions of the IGM with similar matter density. As a first example application, we apply our findings to constrain ALPs (Section 4) and summarize our results (Section 5).

\section{METHODS}

\subsection{Simulations}

IllustrisTNG (TNG) is a suite of large-volume cosmological gravomagnetohydrodynamic simulations (Nelson et al. 2018; Springel et al. 2018; Pillepich et al. 2018b; Naiman et al. 2018; Marinacci

1 This effect places constraints on the IGMF from gamma-ray observations, as mentioned above 

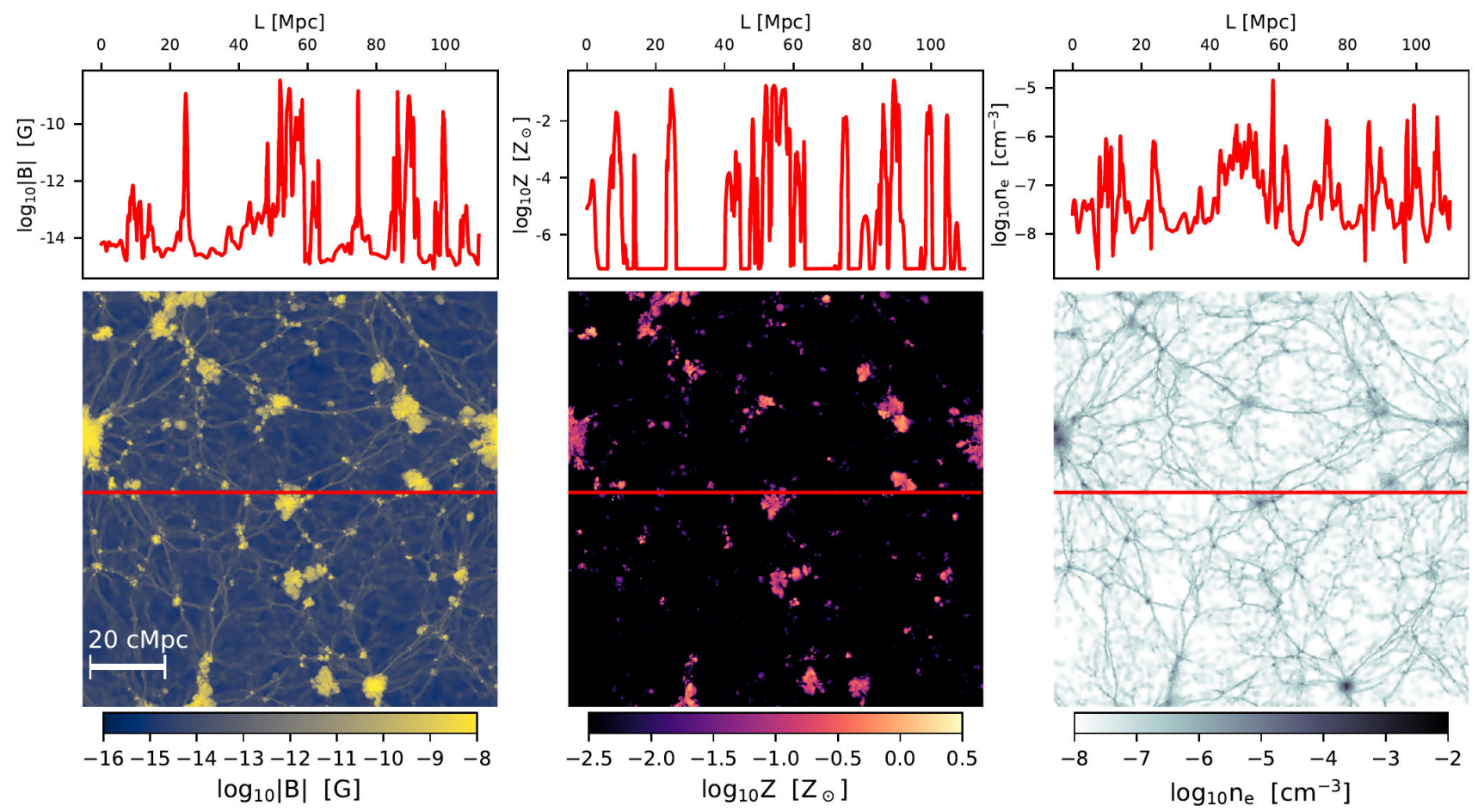

Figure 1. Lower panels show distributions of magnetic field, metallicity and electron number density for a thin slice of the the whole $z=0$ TNG100 box, $(110 \mathrm{Mpc})^{2} \times 20 \mathrm{kpc}$. The upper panels show values of corresponding quantities along one particular line of sight (indicated by the red line on the lower panels). Significant variation occurs as sightlines pass through underdense versus overdense regions.

et al. 2018). Each evolves initial conditions from $z=127$ to the present time, following the evolution of gas, stars, and black holes (baryons), together with dark matter. The TNG simulations use the moving-mesh AREPO code (Springel 2010) to solve the coupled equations of self-gravity and ideal MHD (Pakmor et al. 2011; Pakmor \& Springel 2013), and adopt cosmological parameters consistent with Planck 2015 (Planck Collaboration et al. 2016). The simulations include a comprehensive galaxy formation model incorporating astrophysical processes such as gas metal-line cooling and heating, star formation, stellar evolution, and heavy element enrichment, supermassive black hole growth, AGN feedback, and galactic winds launched by supernovae (Weinberger et al. 2017; Pillepich et al. 2018a). The TNG project currently spans three different volumes, TNG50, TNG100, and TNG300, each run with several different numerical resolutions. In this work, we mainly use the publicly available TNG100-1 simulation (Nelson et al. 2019a), the highest resolution run of TNG100, with a box side-length of $L \sim 100 \mathrm{cMpc}$ (comoving Mpc). Containing $1820^{3}$ dark matter particles and an equal number of gas cells, it has a mass resolution of $m_{\text {bar }}=1.4 \times 10^{6} \mathrm{M}_{\odot}$, and $m_{\mathrm{DM}}=7.5 \times 10^{6} \mathrm{M}_{\odot}$, respectively. From now on, we refer to such simulation as TNG100.

\subsection{Galaxy formation model}

As we are particularly interested in the role of galactic-scale outflows in producing extended regions of high magnetic field strength, we describe the feedback physics briefly. A supermassive black hole (SMBH) is created in all dark matter halos which exceed a total mass of $\sim 7 \times 10^{10} \mathrm{M}_{\odot}$, by placing a SMBH at the potential minimum with an initial mass of $\sim 10^{6} \mathrm{M}_{\odot}$. These black holes sub- sequently grow via binary mergers with each other during galaxy collisions, and via smooth gas accretion from the surrounding environment. Black hole accretion is calculated using the Bondi-HoyleLyttleton assumption (Weinberger et al. 2017), which depends on the black hole mass, local gas density, and relative velocity between the black hole and its surroundings. The accretion rate onto SMBHs is limited to the Eddington rate. To model energetic feedback from SMBHs, a small fraction of the rest mass of accreted matter is available to be deposited back into the locally surrounding gas. This energy is injected in a dual-mode model, depending on this accretion rate: one mode is for the high-accretion state (above $\sim 10$ percent of the Eddington rate), while the second, low-accretion state feedback mode, operates for accretion rates roughly below this value.

At high accretion rates, energy is deposited in a continuous manner, by thermally heating gas. At low accretion rates, kinetic energy is injected in a discrete rather than continuous fashion, such that feedback events occur once enough energy accumulates (see Weinberger et al. 2017 for additional details). In this mode each injection event is modeled as a high-velocity kinetic wind, which is oriented randomly for each event, producing a timeaverage isotropic energy injection. This sub-resolution model is based on theoretical expectations for low accretion rate black holes, i.e. below one percent of Eddington, which develop radiatively inefficient flows and thereby convert gravitational binding energy into a non-relativistic wind (Blandford \& Begelman 1999; Yuan \& Narayan 2014). In the TNG model, it is this population of low luminosity, slowly accreting SMBHs which drive the most powerful outflows (Nelson et al. 2019b). This occurs above a characteristic galaxy stellar mass (dark matter halo mass) of $\sim 10^{10.5} \mathrm{M}_{\odot}$ ( $10^{12} \mathrm{M}_{\odot}$ ), corresponding to the onset of quenching in the 

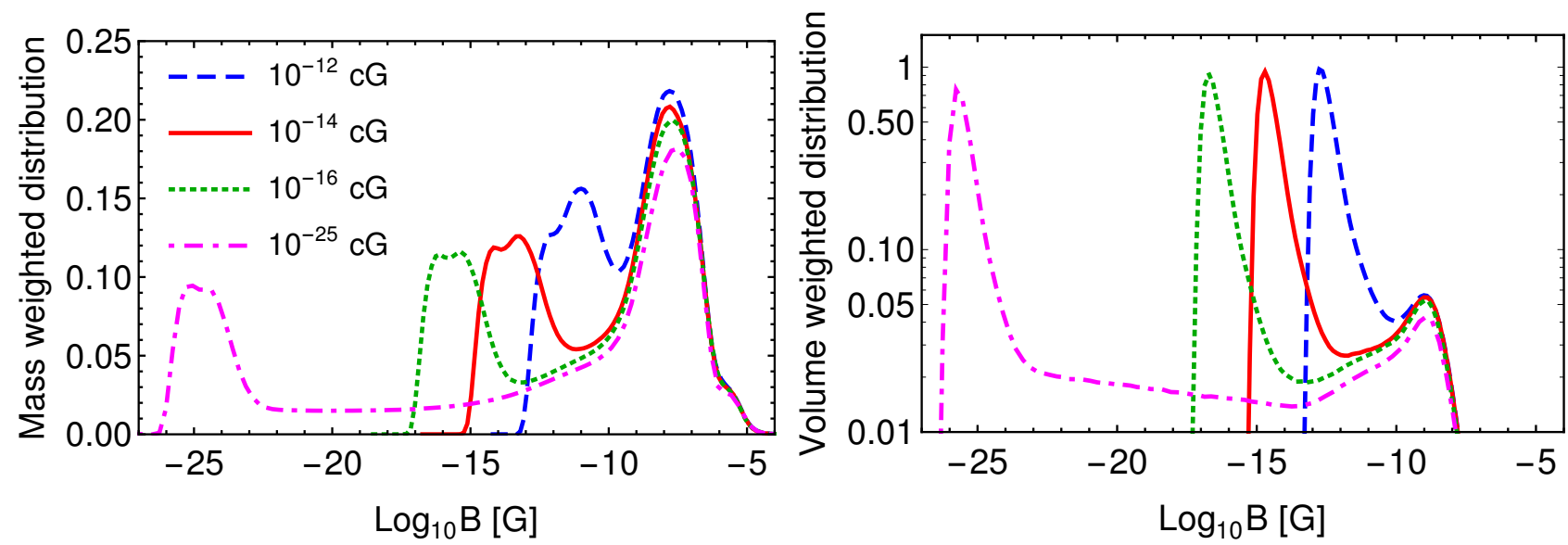

Figure 2. Mass weighted (left panel) and volume weighted (right panel) distributions of the magnetic field strength at $z=0$ for four simulations with different seed field values but with the same underlying TNG galaxy formation model, over $25 \mathrm{cMpc} / \mathrm{h}$ a-side volumes: $10^{-12} \mathrm{cG}$ (blue dashed), $10^{-14} \mathrm{cG}$ (red solid, the fiducial choice of the TNG simulation adopted throughout the paper), $10^{-16} \mathrm{cG}$ (green dotted), $10^{-25} \mathrm{cG}$ (magenta dot-dashed), where cG are comoving Gauss. While the magnitude $|B|$ depends on the assumed initial field strength for very low field strengths, corresponding to underdense/void-like regions of the simulated universe (see Fig. 3), the magnetic field strength within collapsed structures at $\log (|B| / \mathrm{G}) \gtrsim-10$ is largely unchanged, due to the rapid amplification processes which effectively erase knowledge of the primordial seed fields explored in this work.

\begin{tabular}{|l|c|c|c|c||c|c|c|c|}
\cline { 2 - 9 } \multicolumn{1}{c|}{} & \multicolumn{4}{c||}{ Mass weighted fraction } & \multicolumn{4}{c|}{ Vlume weighted fraction } \\
\hline$B_{0}[\mathrm{cG}]$ & $10^{-12}$ & $10^{-14}$ & $10^{-16}$ & $10^{-25}$ & $10^{-12}$ & $10^{-14}$ & $10^{-16}$ & $10^{-25}$ \\
\hline$B>10^{-12} \mathrm{cG}$ & - & $68.6 \%$ & $64.6 \%$ & $57.5 \%$ & - & $14.8 \%$ & $13.8 \%$ & $11.4 \%$ \\
\hline$B>10^{-11} \mathrm{cG}$ & - & $62.8 \%$ & $60.2 \%$ & $53.6 \%$ & - & $12.1 \%$ & $11.4 \%$ & $9.5 \%$ \\
\hline$B>10^{-10} \mathrm{cG}$ & $62.0 \%$ & $57.0 \%$ & $54.8 \%$ & $48.9 \%$ & $9.4 \%$ & $9.0 \%$ & $8.5 \%$ & $7.1 \%$ \\
\hline$B>10^{-9} \mathrm{cG}$ & $50.9 \%$ & $48.3 \%$ & $46.7 \%$ & $42.0 \%$ & $4.6 \%$ & $4.6 \%$ & $4.3 \%$ & $3.6 \%$ \\
\hline
\end{tabular}

Table 1. Fraction of large-value magnetic fields calculated by mass and volume weighted distributions from the $25 \mathrm{cMpc} / \mathrm{h}$ simulations with different seed magnetic field values $B_{0}$ presented in Fig. 2. We excluded values for two fractions for the largest seed magnetic field $10^{-12} \mathrm{cG}$ because they are significantly contaminated by the seed field.

galaxy population (Weinberger et al. 2018; Donnari et al. 2020). Lower mass galaxies can also produce strong outflows, via a model for supernovae-driven winds originating from SNII explosions associated with ongoing star formation (see Pillepich et al. 2018a for more details). In general, these outflows are slower and do not escape to distances as large as black hole-driven outflows (Nelson et al. 2019b).

\subsection{Initial conditions and model variations}

When specifying the initial conditions for the gas, in addition to small density and velocity perturbations required to realize the chosen cosmological constraints, TNG must also specify the initial conditions for the magnetic fields, which are given in terms of the strength and direction of the initial magnetic fields, which can be very different for the different production mechanisms, i.e. astrophysical and primordial (Garaldi et al. 2020). All TNG simulations to date have been run with the same configuration of the initial magnetic field, which is assumed to be a constant volume-filling field (which is an approximation for a primordial, i.e. inflational magnetogenesis, and which is the common practice in such simulations. In this work we mainly use the TNG100 box with the initial strength $B_{0}=10^{-14} \mathrm{cG}$ (comoving Gauss). At $z=127$ this corresponds to a physical field strength of $B_{0}=0.16 \mathrm{nG}$.

In addition, a large number of 'TNG model variation' simulations have been run, each changing a single parameter value or model choice to assess its importance in the fiducial TNG galaxy formation model (Pillepich et al. 2018a). These simulate smaller $25 \mathrm{cMpc} / \mathrm{h}$ volumes, each realized at three resolution levels equivalent to those available for TNG100 itself. In this work, we primarily use four simulations which vary the initial magnetic field strength, adopting $B_{0}=10^{-25} \mathrm{cG}, 10^{-16} \mathrm{cG}, 10^{-16} \mathrm{cG}$ (the fiducial choice of the TNG100 flagship run) and $10^{-12} \mathrm{cG}$. We also inspect the outcome of three other runs that, compared to the TNG fiducial model, include no SMBH feedback, no SMBH kinetic feedback, and no feedback of any type, respectively.

\subsection{Analysis methodology}

We analyze these simulations from $0 \leqslant z \leqslant 6$ and measure the density of free electrons and magnetic field strength, avoiding $z>6$ where these quantities become uncertain during the epoch of reionization (Heinrich et al. 2017; Aghanim et al. 2020). The electron number density is calculated from the helium and hydrogen number densities and their ionization states. We use the spectral synthesis code CLOUDY V17.01 (Ferland et al. 2017) to calculate the ion fractions of hydrogen and helium for gas exposed to a redshift-dependent UV background (Haardt \& Madau 2012). We neglect the contribution of ionization states from elements heavier than $\mathrm{He}$, as well as molecular gas phases.

The magnetic field and electron number density are smoothed 
onto a grid with $(20 \mathrm{ckpc})^{3}$ voxels. $^{2}$ One thousand lines of sight (LOS) are generated for each available snapshot with random orientation within the simulated volume, and we use these throughout as our fiducial set of sightlines. In Fig. 1 we show an example of the magnitude of the magnetic field, metallicity, and electron number density image of a given region together with a potential LOS. The regions of magnetic field enhancement extend beyond gravitationally collapsed structures (i.e. dark matter halos), c.f. also Fig. 7. They also extend, in some cases, to substantially larger distances, due to the ejection of magnetic fields in supernovae and black holedriven galactic outflows (Nelson et al. 2019b).

\section{STATISTICAL PROPERTIES OF SIMULATED MAGNETIC FIELDS}

We start by examining the three $25 \mathrm{cMpc} / \mathrm{h}$ variation boxes with different initial magnetic field seed conditions. In particular, we aim to determine to what extent the predicted IGMF depends on the value of the initial seed. In Fig. 2 (left panel) we show the mass-weighted distribution of the magnetic field for three different choices of the initial field. These global distributions across all gas cells contain two clear peaks - a low- $B$ peak that has its center at the value of the initial field, and a strong- $B$ peak, that are very similar for all four values of the initial conditions.

It is important to keep in mind that the number of gas cells with a given value of the field does not represent the fraction of volume occupied by such a field. As the simulation is spatially adaptive and maintains a constant mass resolution, gas cells accumulate inside high-density regions. We therefore also show the volume-weighted distribution of magnetic field strength in Fig. 2 (right panel). Both cases show the same picture: that the high- $B$ component of the distribution is weakly sensitive to the magnitude of the initial field. To characterize difference in strong- $B$ peaks numerically we calculated mass and volume weighted fractions within this peaks for different seed fields, see Table 1 . We see that while the seed field value changes by 13 orders of magnitude, all fractions change by less then a factor 1.3 . This is due to the rapid amplification processes which effectively erase knowledge of the primordial seed fields (see also Pakmor et al. 2014b) explored in this work. Note that these distributions include not only galaxies and clusters but also larger volumes potentially affected by outflows, and the surrounding intergalactic medium, as we discuss in more details below.

To study the large-value component of the magnetic field more quantitatively we analyze the main simulation box TNG100 with a seed magnetic field $10^{-14} \mathrm{cG}$. Let us consider its distribution versus electron number density at different redshifts, shown in Fig. 3 (see also Marinacci et al. 2018). At low redshift $z \lesssim 2$ we see two distinct branches, corresponding to weak and strong magnetic fields, respectively. In both branches, the value of the magnetic field is correlated with the electron number density. Even in the regions with small values of electron number density $\sim 10^{-8} \mathrm{~cm}^{-3}$ (onetenth of the average electron number density today), the magnetic field can be many orders of magnitude stronger than its average value (close to the value of the initial field). This strong- $B$ (or "over-magnetized") branch in Fig. 3 corresponds to the primordial seed independent strong- $B$ peak in Fig. 2. In Appendices A, B, C

2 We use the publicly available pysph-viewer code (Benitez-Llambay 2015) for this deposition. we present plots similar to Fig. 3 that illustrate that two branches also exist for $z<2$ for different initial conditions and different box sizes, while for $z>2$ there is generically only one branch in the $B-n_{e}$ plot, due to the time needed for exponential amplification via a small-scale turbulent dynamo. This amplification process is faster at higher numerical resolution, enabling the magnetic fields to reach their quasi-saturated values earlier, although the level of this final saturation is relatively unaffected by resolution (Pakmor et al. 2017). In such a relatively small volume, there are also no high-mass halos at early times $(z \gtrsim 3)$, such that large outflowdriven bubbles have not yet formed. Either larger volumes, i.e. probing the environments of the largest overdensities, or higher resolution would therefore if anything enhance the importance of these structures.

Our goal is to understand the impact of the outflow-generated large- $B$ component of the magnetic field distribution on the propagation of light through the Universe. Specifically, what is the probability for a photon to occupy a large- $B$ region on its way towards an observer? Fig. 4 therefore shows the volume fraction of regions with large magnetic field values, excluding the regions where the electron concentration is larger than some given value (in this way we can, in particular, exclude the inner parts of the collapsed structures like galaxies). ${ }^{3}$ At $z=0$ in regions with $n_{e}<100\left\langle n_{e}\right\rangle$ the magnetic field is stronger than $10^{-12} \mathrm{cG}$ in $\sim 14 \%$ of the volume, while it is stronger than $10^{-10} \mathrm{cG}$ in $\sim 8 \%$ of the volume. We note that the value of the initial field is $10^{-14} \mathrm{cG}$. Moving only to $z=0.5$, we see that strong- $B$ regions occupy half as much volume, indicating that strong- $B$ regions are substantially enhanced by late time processes.

Alternatively, we can measure the fractional length, for a given line of sight, which intersects a strong magnetic field. Using our sample of 1000 random sightlines we show in Fig. 5 the distribution of length fractions having magnetic field strength $B \geqslant 10^{-12} \mathrm{cG}$ at four different redshifts. ${ }^{4}$ At $z=0$ more than half of all sightlines intersect such strong magnetic fields along more than $10 \%$ of their path length. The peak of the distribution shifts to smaller fractional path lengths towards higher redshift. At $z \geqslant 1$, sightlines intersect magnetic fields of this strength only rarely.

For further applications, it is also interesting to consider longer lines of sight that do not fit into one $\sim 100 \mathrm{cMpc}$ snapshot. Using the TNG simulation volume we constructed continuous lines of sight from $z=0$ to $z=6$ following the procedure described in Bondarenko et al. (2020). To construct the magnetic field along a continuous line of sight we take $B(z)$ from additional random sightlines within the same snapshot, and assign it to any missing pathlength (between simulation snapshots) of the continuous LOS, rescaling as $B \propto(1+z)^{2}$. An example of both electron number density and magnetic field strength along a single continuous sightline is shown in Fig. 6.

\subsection{Connection between over-magnetized 'bubbles' and galaxy outflows}

Visual inspection of Fig. 1 reveals the strong connection between over-magnetized bubbles (i.e. the regions where magnetic fields are orders of magnitude larger than in the average regions with the

3 For similar figures for different seed field values and box sizes see Figs. A2, B2, and C2.

4 For similar figures for different seed field values and box sizes see Fig. B3. 


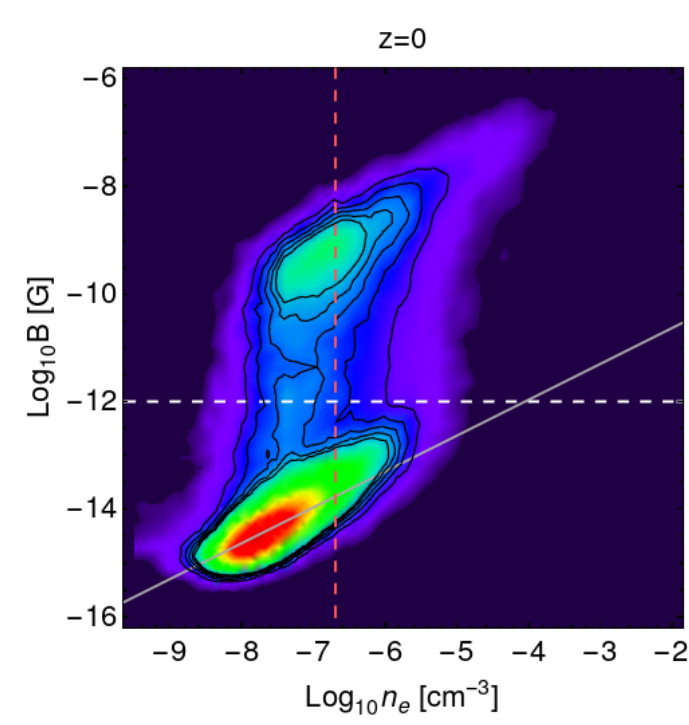

$z=1$

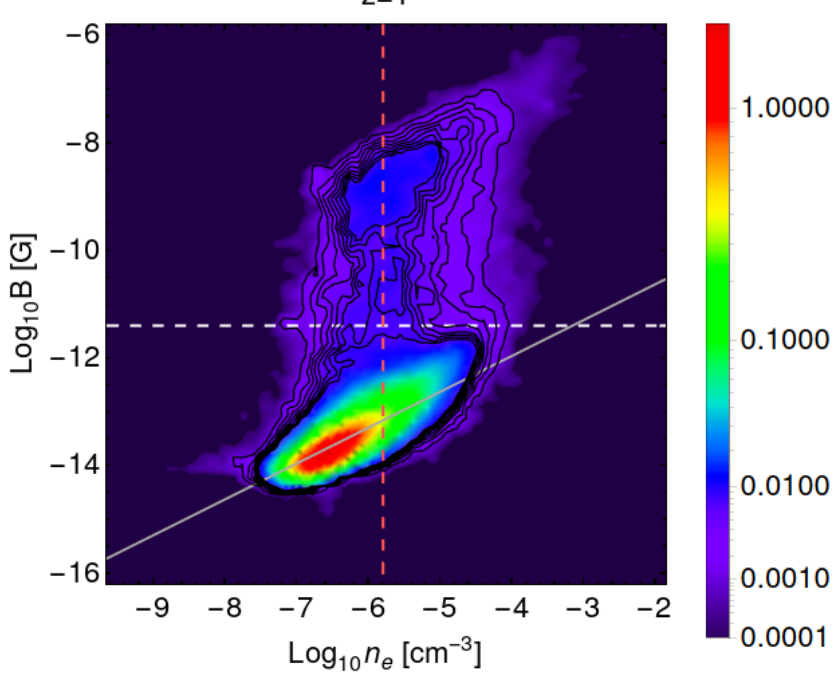

PDF

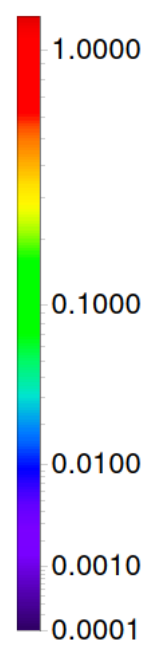

PDF

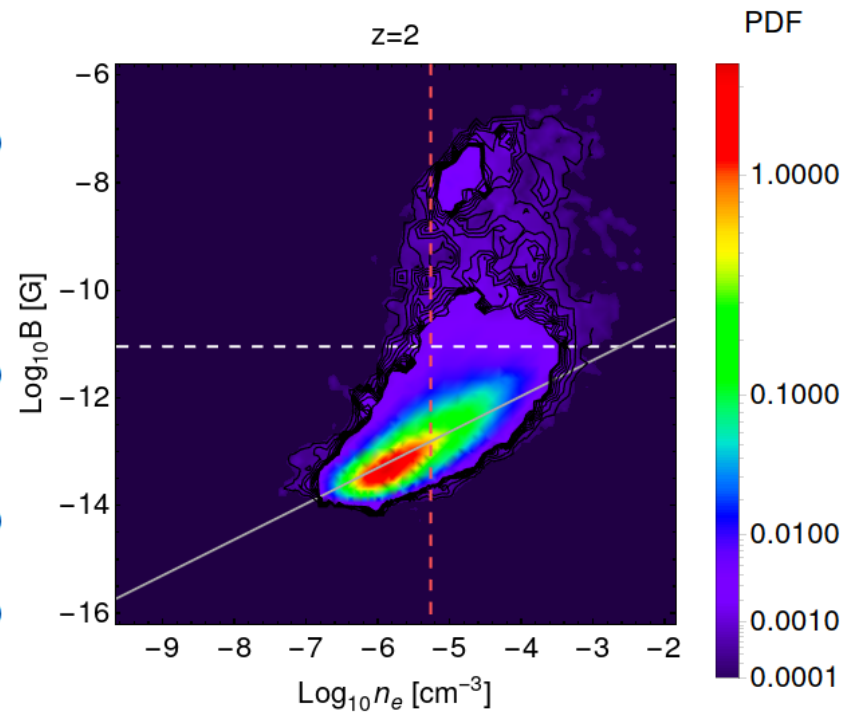

PDF

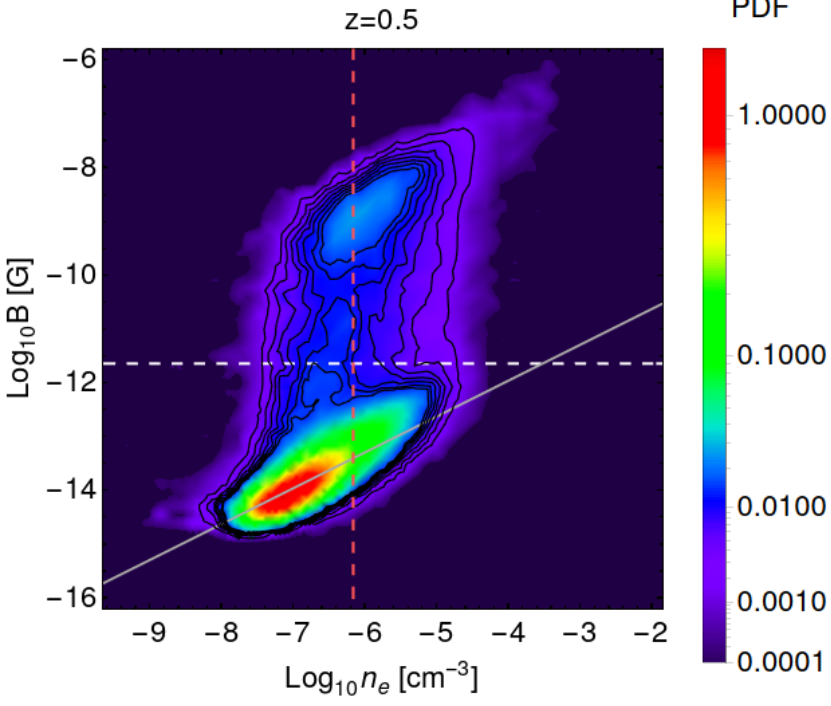

.1000

0100

0001

DF

0000

Figure 3. Distribution of the magnetic field strength and electron number density in the TNG100 simulation using data along 200 random lines of sight with $(20 \mathrm{ckpc})^{3}$ voxels in the box (see Sec. 2.4 ), where the seed field is $B_{0}=10^{-14} \mathrm{cG}$, at redshifts $z=0,0.5,1$ and 2 . The color indicates the occupied volume fraction. The dashed white line corresponds to the comoving magnetic field value $10^{-12} \mathrm{cG}$ that we use as the smallest value of outflow-generated magnetic fields in this work. The red dashed line represents the average electron number density at a given redshift. The gray dashed line shows a power law $B \propto n_{e}^{2 / 3}$ that represents adiabatic evolution (see e.g. Durrer \& Neronov 2013). At fixed electron number density, two distinct branches of magnetic field strength are apparent, corresponding to weak and strong components, respectively. Results for other redshifts and simulation boxes are given in Appendices A, B, and C.

same values of $n_{e}$, see Fig. 3 and discussion), metallicity, and the structures seen in the electron number density. This is consistent with the behavior seen in the TNG galaxy formation model where strong outflows can escape from galaxies and break out into the intergalactic medium, carrying mass, heavy elements, and magnetic energy density along the way (Nelson et al. 2019b). This behavior is also consistent with results for galactic and near-galactic magnetic fields discussed in Butsky et al. (2016); Pakmor et al. (2020); Martin-Alvarez et al. (2020).

To explore the physical connection between large-scale overmagnetized 'bubbles' and galactic-scale feedback processes, Figure 7 shows the magnetic field strength in thin $(20 \mathrm{kpc})$ slices of TNG100 at $z=0$. In the left panel, we mark all dark matter halos with total mass $>10^{11.5} \mathrm{M}_{\odot}$ with red circles, where the marker size denotes $1.5 R_{200}$, where $R_{200}$ is the virial radius of a halo. In the right panel, we instead mark all supermassive black holes which have injected a significant amount of energy in the lowaccretion state, $E_{\text {low }}>10^{58.5} \mathrm{erg}$, with stars. We see that most magnetized bubbles extending to $\gtrsim$ Mpc scales are directly associated with massive halos and/or supermassive black holes near their center.

This is, however, not always the case. Figure 8 zooms into a crowded region of the same slice, within which an over-magnetized bubble is evident. The three panels on the right show magnetic field strength, gas metallicity, and electron number density. There is no clear association between cosmic web filaments visible in $n_{e}$ and regions of strong $|B|$. We have specifically selected this region as having no supermassive black holes which satisfy our energy criterion. Their absence implies that either the SMBH sourcing this bubble is outside the $\pm 2.5 \mathrm{Mpc}$ vicinity of the slice or that this large bubble may be a collective effect of galactic-scale winds produced by ongoing supernovae explosions in lower mass, 

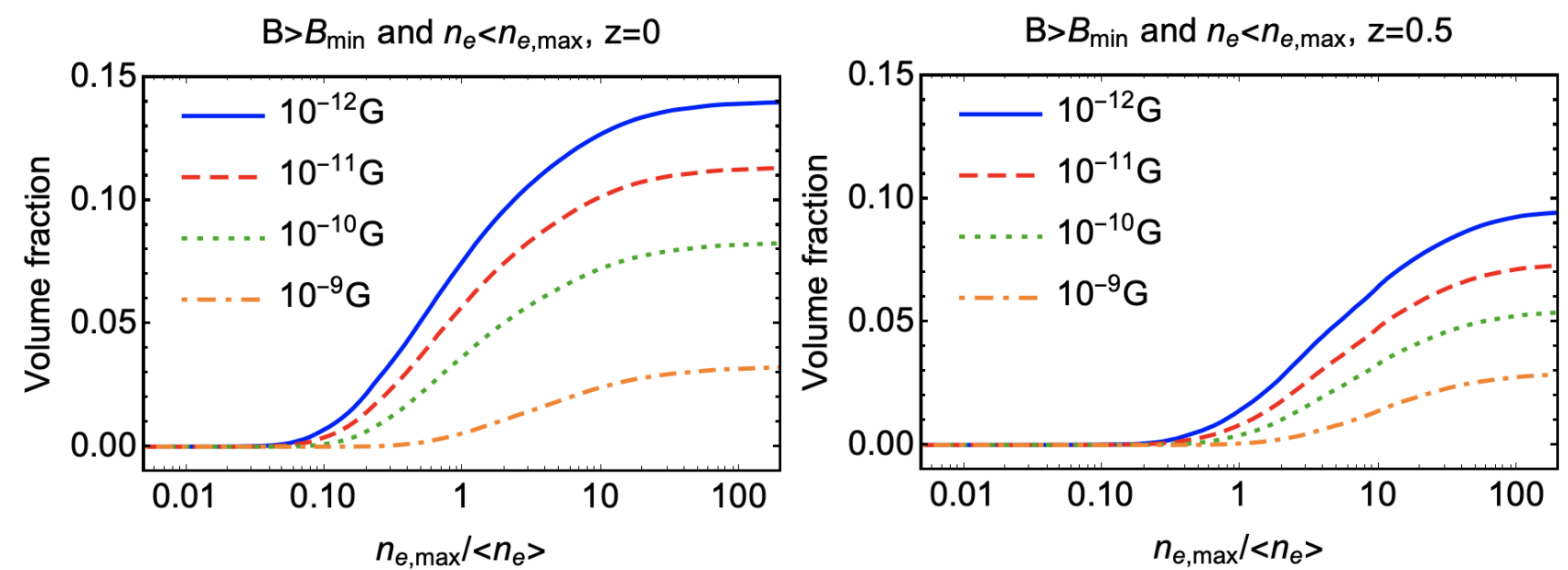

Figure 4. Volume fractions of the regions where the magnetic field is larger than $B_{\min }$ (legend) and the electron number density is smaller than $n_{e, \text { max }}$ (x-axis) for $z=0$ (left panel) and $z=0.5$ (right panel). We here show TNG100 with a seed field of $B_{0}=10^{-14}$ cG using data along 200 random lines of sight with $(20 \mathrm{ckpc})^{3}$ voxels in the box (see Sec. 2.4). Comparing these two redshifts, a substantial growth of the volumes occupied by high magnetic field strengths is evident, implying that physical processes within the last 5 Gyr have had a strong impact. Results for other redshifts and simulation boxes are given in Appendices A, B, and C.
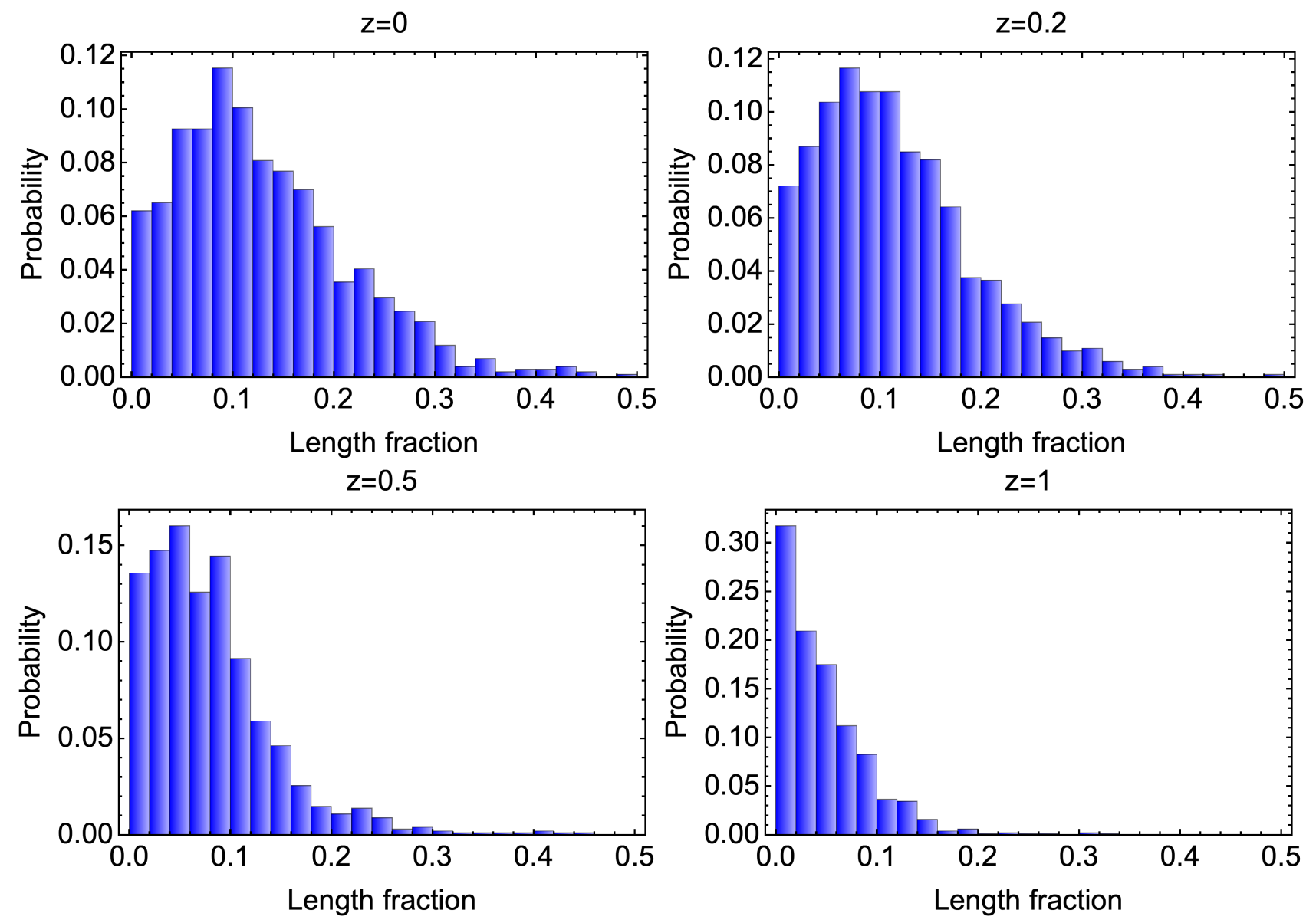

Figure 5. Probability to find a fraction of length along the line of sight with magnetic field larger than $10^{-12}$ comoving Gauss. We show results for TNG100 at redshifts $z=0,0.2,0.5$, and 1 , creating 1000 random lines of sight at each redshift. While there is negligible 'strong magnetic field path length' at high redshifts, by $z=0$ roughly half of all sightlines intersect such strong magnetic fields along more than 10 percent of their length. Results for other redshifts and simulation boxes are given in Appendices B and C. 

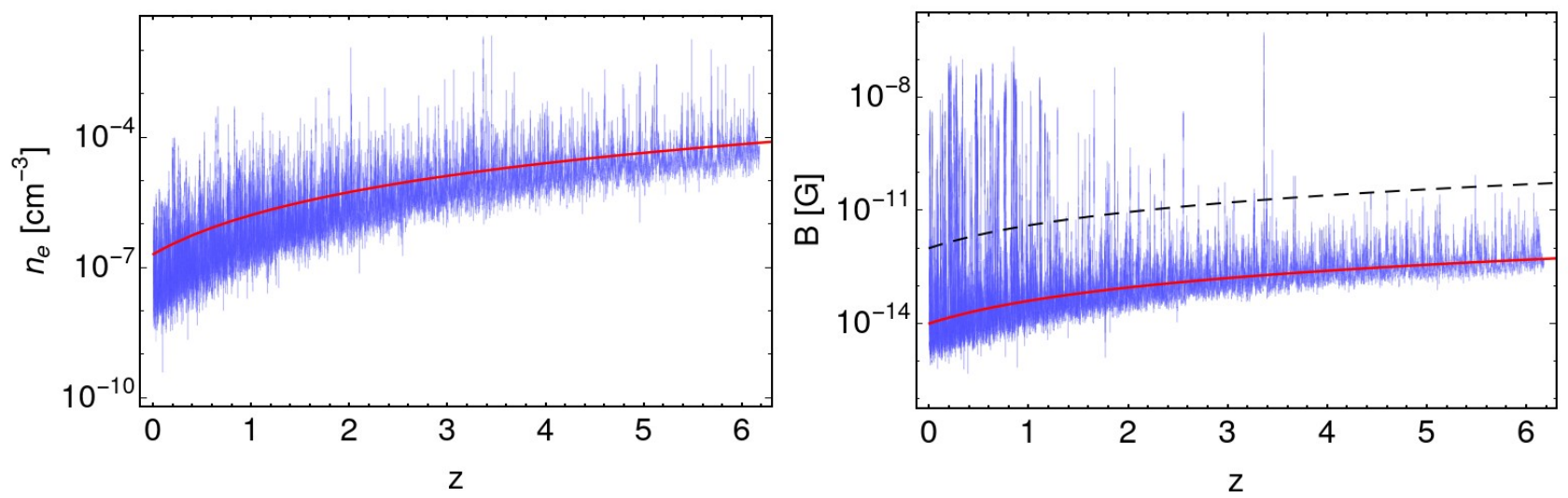

Figure 6. An example along a single continuous line of sight through the TNG100 simulation: electron number density (left panel) and magnetic field strength (right panel). The red line on the left panel shows the average electron number density in the Universe. The red line on the right panel corresponds to the seed value of the magnetic field, while the black line shows $B=10^{-12} \mathrm{cG}$.
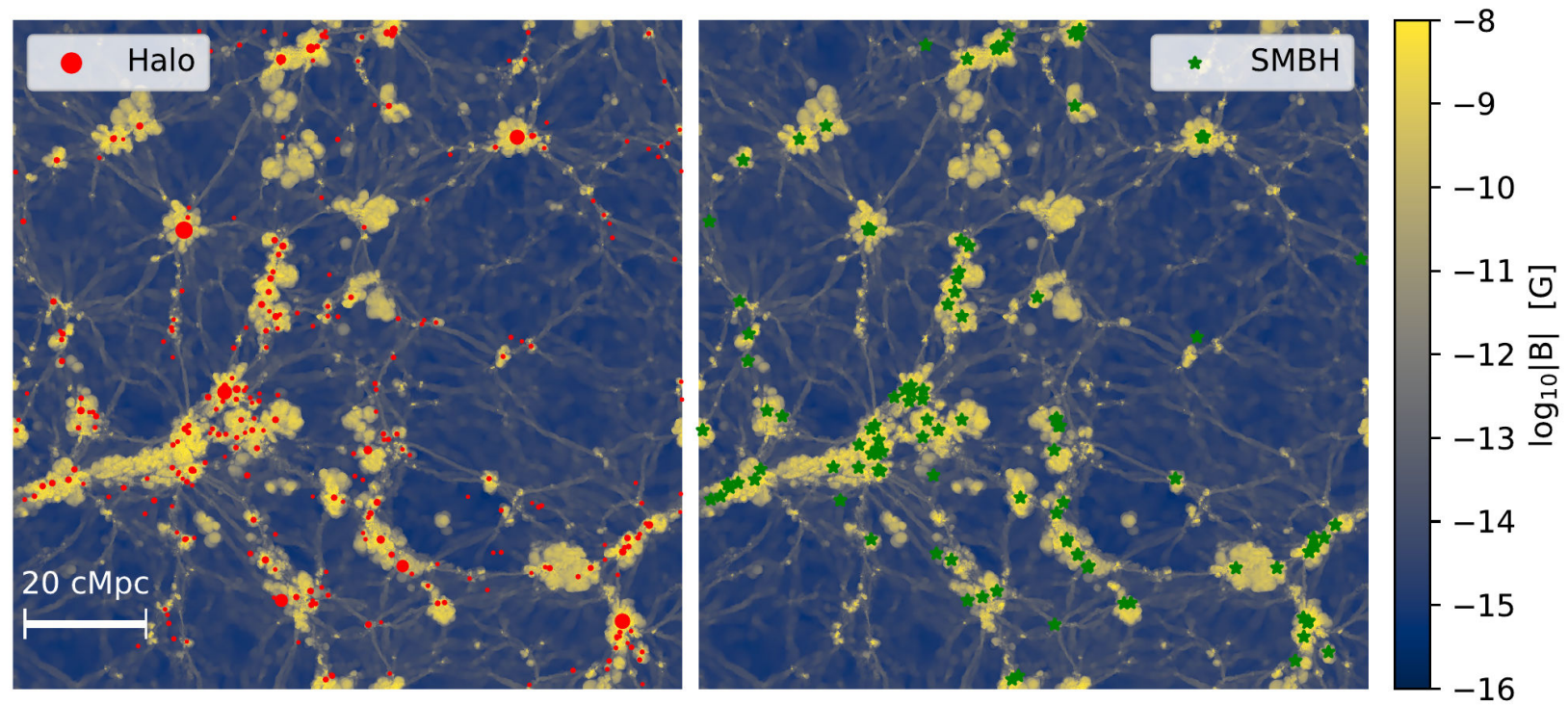

Figure 7. The magnetic field distribution for the same slice as in Fig. 1, from the TNG100 simulation. Within $\pm 2.5 \mathrm{Mpc}$ of this slice we indicate all halos with mass $10^{11.5} M_{\odot}$ and above (red circles; left panel, radii corresponding to $1.5 \mathrm{R}_{200}$ ) and all black holes (stars; right panel) which have injected significant amount of energy back to the gas (with $E_{\text {low }}>10^{58.5} \mathrm{erg}$, see discussion in the text). Both are strongly correlated with the presence of large-scale magnetized bubbles.

star-forming galaxies, possibly in combination with the effect of a number of smaller black holes.

To better understand the origin of these bubbles, Fig. 9 shows the magnetic field, gas temperature, dark matter, and electron number densities in a region extending $12 \mathrm{Mpc} \times 14.1 \mathrm{Mpc} \times 15 \mathrm{Mpc}$ that contains the same large over-magnetized bubble from the Fig. 8. In this volume, we see a clear filament of large-scale structure. We again mark the massive halos and energetic black holes. Particularly clear around the SMBHs in the center and upper right are signatures of a collimated, episodic outflow made up of successive gas shells, forming a butterfly-like morphology, which results from the SMBH kinetic wind feedback. Here the ejection of magnetic fields into these bubbles is directly caused by active SMBHs.

However, stellar feedback can also contribute, most notably through core-collapse supernovae ( $\mathrm{SN}$ ) explosions. These also produce galactic-scale outflows, albeit in lower mass galaxies. To isolate these two mechanisms, we turn to additional simulations of the 'TNG model variation' suite. In particular, Fig. 10 compares the fiducial model (upper left) to models with no SMBH kinetic wind feedback (lower left), no SMBH feedback of any kind (upper right), and no feedback whatsoever from either black holes or supernovae (lower right). In all cases, the identical $25 \mathrm{cMpc} / \mathrm{h}$ volume is shown. The regions present in the fiducial run, but missing when the kinetic winds are disabled, are due to the low-accretion feedback mode of the SMBHs. The overall extent of the central filamentary region is such an example. Furthermore, regions present in the runs without black hole feedback, but missing in the 'no feedback' case, are due to supernovae - some of the smaller bubbles towards the up- 


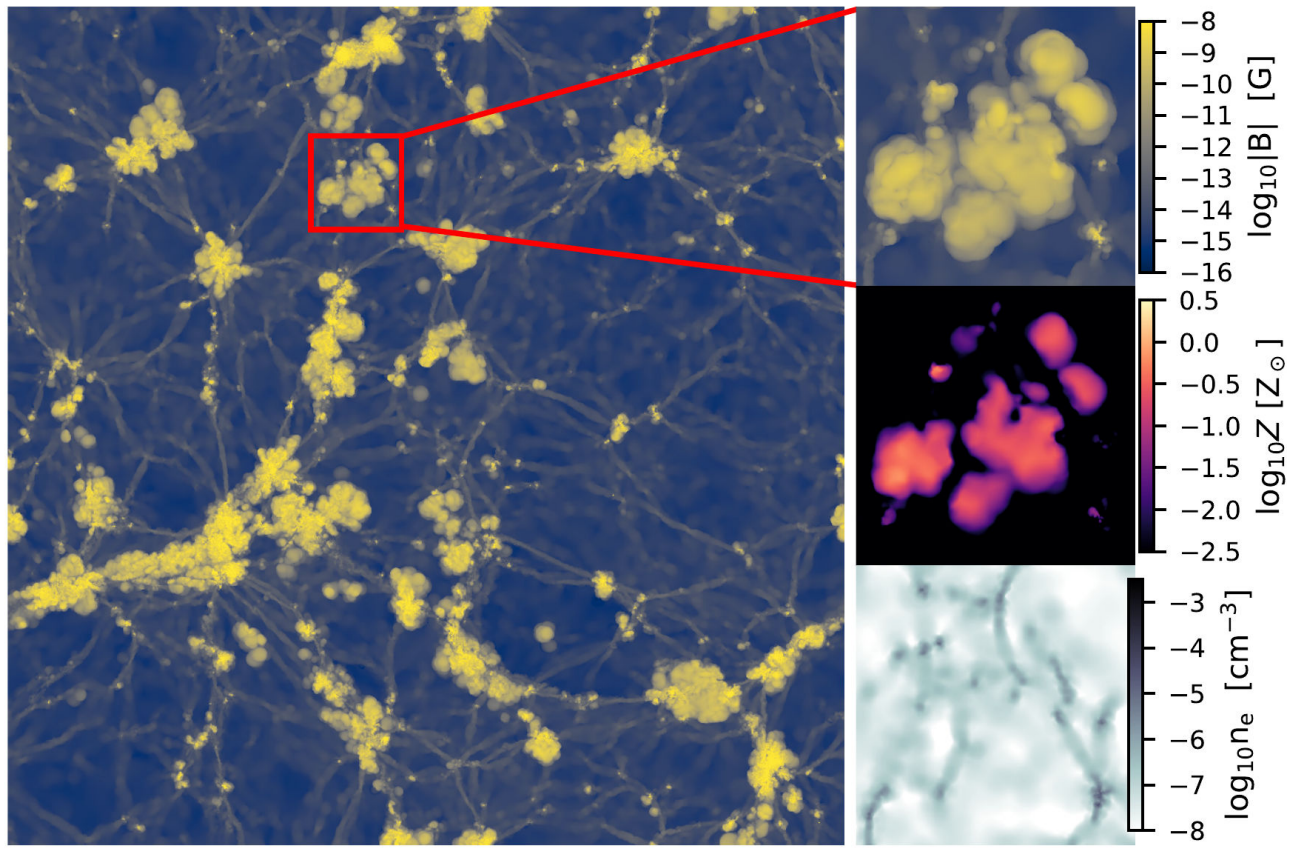

Figure 8. Zoom on a magnetized bubble region from TNG100 selected to have no supermassive black holes satisfying the energy threshold, $E_{\text {low }}>$ $10^{58.5} \mathrm{erg}$, within $5 \mathrm{Mpc}$ of the slice. Further, this region is not clearly associated with an overdensity in $n_{e}$, implying that some large-scale magnetized bubbles can arise from the combined action of many lower mass galaxies, hosting less effective black holes and/or supernovae-driven outflows.

per right being prime examples. In general, bubbles inflated by $\mathrm{SN}$ rather than SMBHs tend to be smaller, have lower temperatures, and lower expansion velocities. Overall, we see that both SMBHs and $\mathrm{SN}$ contribute to extended regions of high magnetic energy, with the supernovae playing a sub-dominant role.

\section{AXION CONVERSION PROBABILITY}

In this section, we apply our findings to calculate the probability of photon-axion conversion during propagation through the IGM. This conversion occurs in the presence of an external magnetic field $B$ due to the interaction term

$$
\mathcal{L}_{a \gamma}=\frac{g_{a}}{4} a F_{\mu \nu} \tilde{F}_{\mu \nu}=g_{a} a\left(\partial_{0} A_{i}\right) \cdot B_{i},
$$

where $g_{a}$ is the ALP constant (with units of inverse energy), $B_{i}$ are components of the magnetic field, and $A_{i}$ are spatial components of $A_{\mu}$ in the gauge $A_{0}=0$. For ALPs with energy $E_{a}$ in an external magnetic field this interaction effectively gives mass-mixing between the ALP and the photon,

$$
\mathcal{L}_{a \gamma}=g_{a} E_{a} B_{i} a A_{i}=g_{a} E_{a} B_{T} a|\boldsymbol{A}|,
$$

where $B_{T}=|B| \cos \theta$ and $\theta$ is an angle between vector $\boldsymbol{A}$ and the magnetic field. This leads to oscillations between axions and photons (Raffelt \& Stodolsky 1988). The strength of the mixing between axions and photons is proportional to the magnetic field as well as the energy of the axion.

The conversion probability also depends on the axion mass $m_{a}$ and the effective photon mass in the medium. For soft enough photons propagating through IGM the effective photon mass is given by (see e.g. Mirizzi et al. 2009)

$$
m_{A}\left(n_{e}\right)=\sqrt{\frac{4 \pi \alpha_{\mathrm{EM}} n_{e}}{m_{e}}}
$$

where $m_{e}$ is the electron mass, $\alpha_{\mathrm{EM}}$ is the fine-structure constant and $n_{e}$ is a free electron number density. It should be noted that for gamma-ray photons this description of the effect on the effective properties of photons may not be sufficient (see e.g. Dobrynina et al. 2015). However, in this paper we deal with less energetic photons and therefore we can use Eq. (3) throughout.

In the case when the effective photon mass is equal to the ALP mass, $m_{a}=m_{A}\left(n_{e}\right)$, the conversion becomes resonant and the conversion probability significantly increases. The conversion probability for this case is (Mirizzi et al. 2009)

$$
P_{a \rightarrow A}=1-p, \quad p=\exp \left(-\frac{\pi E_{a} B_{T}^{2} g_{a}^{2}}{m_{a}^{2}} R\right)
$$

where $R=\left|d \log m_{A}^{2} / d \ell\right|_{\ell=\ell_{\text {res }}}^{-1}, E_{a}$ is the axion energy, $B_{T}$ is the component of the magnetic field orthogonal to the line of sight (direction of axion propagation), $\ell$ is the distance along the line of sight, and the derivative for the $R$ factor is calculated at the point of the resonance. The case $p \ll 1$ is called the adiabatic limit and the conversion probability is close to one, while the case $p \approx 1$ is called the non-adiabatic limit and the conversion probability is given by

$$
P_{a \rightarrow A}^{\text {non-adiab }}=\frac{\pi E_{a} B_{T}^{2} g_{a}^{2}}{m_{a}^{2}} R .
$$

Let us assume that the total conversion probability $P_{\text {tot }}$ during axion propagation along the LOS is much smaller than one. In this case the total conversion probability is given by

$$
P_{\mathrm{tot}} \approx \sum_{i} P_{a \rightarrow A, i}^{\mathrm{non}-\mathrm{adiab}}=\frac{\pi E_{a} g_{a}^{2}}{m_{a}^{2}} \sum_{i}\left(1+z_{i}\right) B_{T, i}^{2} R_{i},
$$

where $E_{a}$ is the axion energy at $z=0$, and the sum is taken for resonances along the line of sight: $z_{i}$ is the redshift and $B_{T, i}$ is the 

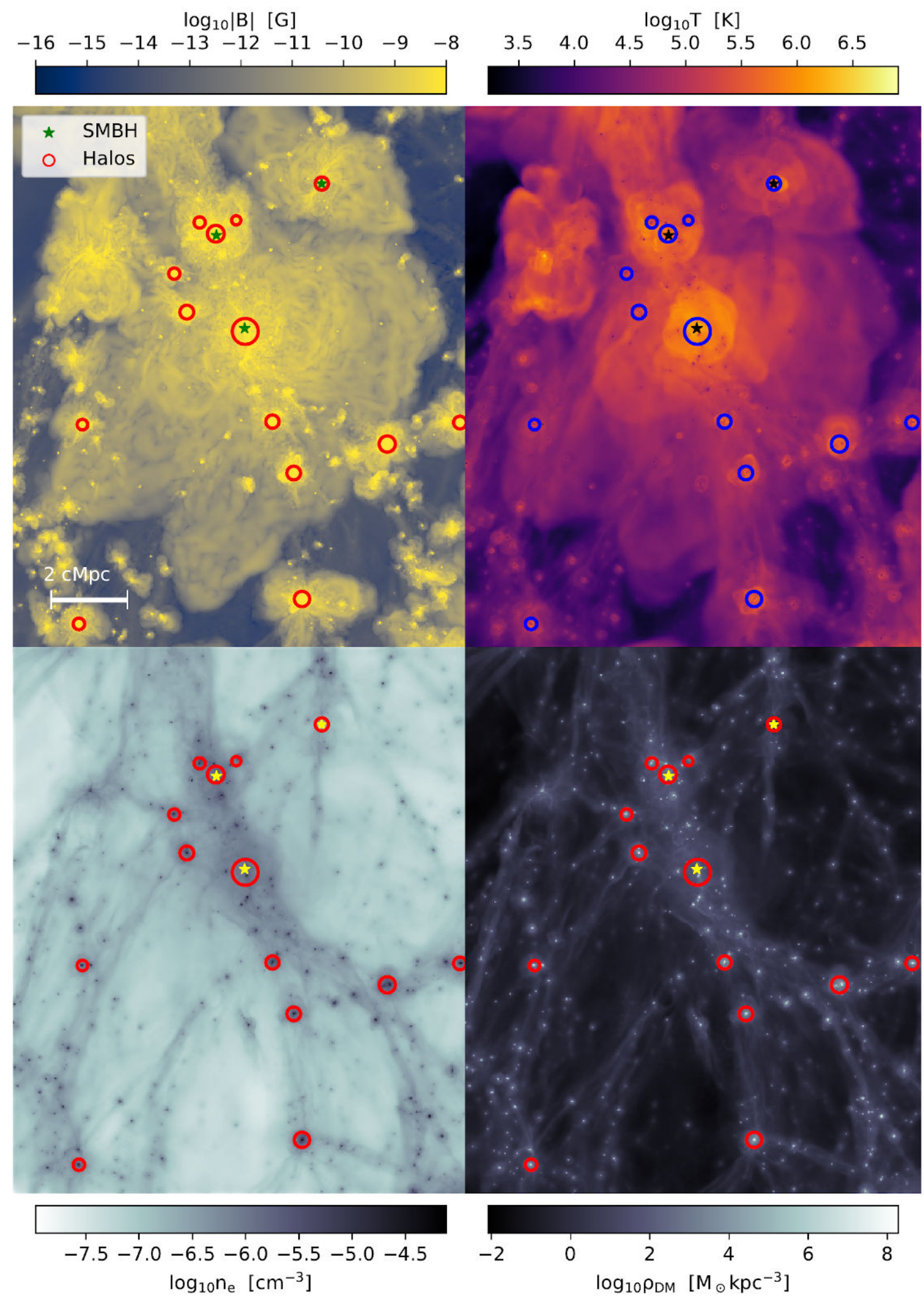

Figure 9. View of a rich, high-overdensity structure from TNG100 which hosts a large-scale, $\sim 10 \mathrm{Mpc}$, outflow-driven bubble that corresponds to the same large over-magnetized bubble from Fig. 8. The top-left panel shows magnetic field strength, next to it the gas temperature. The bottom-left panel shows electron number density and the in the bottom-right we have dark matter density in the region. The three most active supermassive black holes, marked by stars, are largely responsible for the extent of over-magnetized regions. 
Fiducial

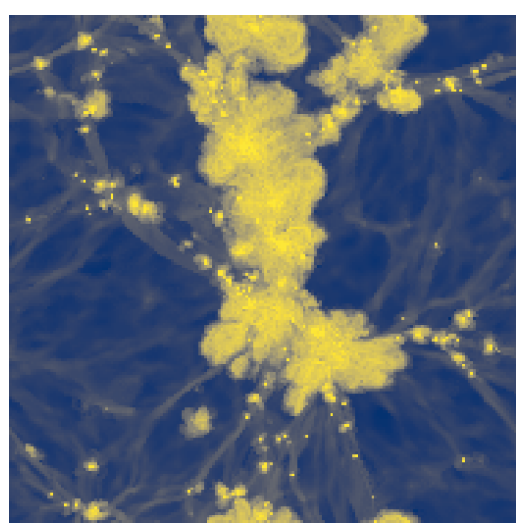

$E_{\text {low }}$ feedback off

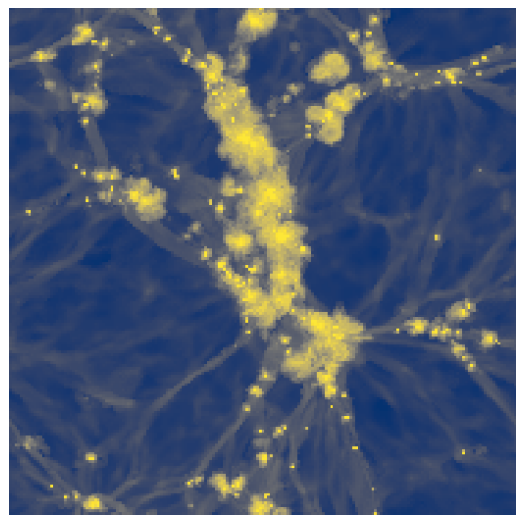

SMBH feedback off

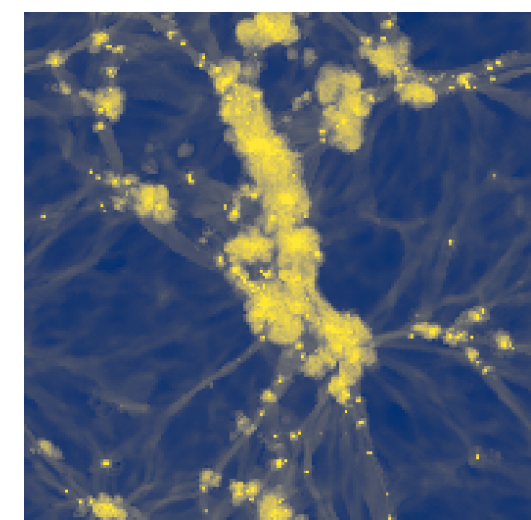

Feedback \& metal cooling off

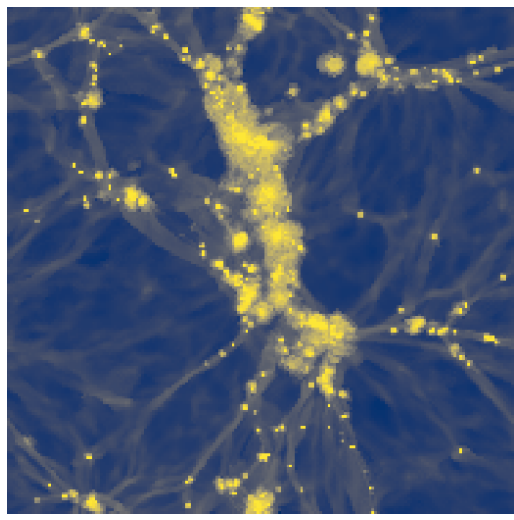

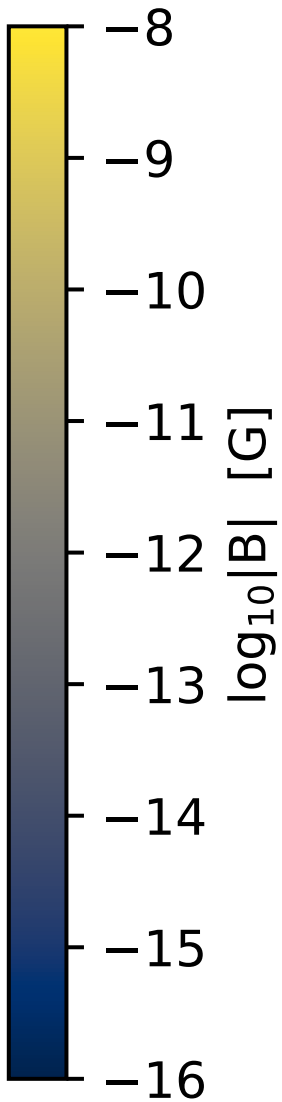

Figure 10. A $(25 \mathrm{Mpc})^{2} \times 1 \mathrm{Mpc}$ slice of the magnetic field from four $25 \mathrm{cMpc} / \mathrm{h}$ simulations at $z=0$. We contrast the fiducial TNG model (upper left) to a variation with no black hole feedback of any kind (upper right), only SMBH low-state kinetic winds disabled (lower left), and no feedback from black holes nor supernovae at all (lower right). In each variation panel there are fewer and smaller large-scale magnetic bubbles with respect to the fiducial model, with the efficient SMBH kinetic wind mode playing a dominant role.

component of the magnetic field orthogonal to the line of sight for a given resonance.

\subsection{Probability of axion conversion along random sightlines}

As the mixing strength between axions and photons is proportional to the magnetic field strength squared, the probability of the axionphoton conversion is dominated by the contribution of the strong- $B$ component of the IGMF distribution. As we have seen above, this part of the distribution is to a large extent universal - its dependence on the value of the initial seed field is negligible (at least within the IllustrisTNG framework and within the class of initial conditions used in this paper). Further study of the dependence on initial conditions from a wider class (e.g. those of Vazza et al. 2017) and with different models of baryonic physics will be considered in future work.

We proceed with our analysis based on the TNG simulations with initial magnetic field strength $10^{-14} \mathrm{cG}$. We take into account only the contribution to conversion from the simulation pixels with the value of magnetic field $B \geqslant 10^{-12} \mathrm{cG}$ (see the black line in Figs. 3 and 6). As we discussed in Section 3, for such magnetic field values, the distribution of magnetic field only slightly depends on the properties of the primordial seed field for seed fields $10^{-14} \mathrm{cG}$ and below. As a result, this threshold on magnetic field strength (in comoving units) returns a somewhat conservative contribution to the axion conversion probability from the magnetic bubbles.

Using 500 simulated continuous lines of sight (as described in Section 2.4), we calculate the average conversion probability and its distribution between redshifts $z=0$ and $z=6$. The result as a function of resonant electron number density is shown in Fig. 11. We see that conversion probability is maximal near $n_{e, \text { res }}=10^{-5} \mathrm{~cm}^{-3}$. The scatter grows for large and small values of $n_{e, \text { res }}$ because of the small amount of resonances along the line of sight. In the region of resonant electron number densities below $10^{-8} \mathrm{~cm}^{-3}$ or above $10^{-2} \mathrm{~cm}^{-3}$ the resonant condition occurs rarely (see Fig. 6). We therefore derive below the constraints on the axion-photon coupling for the axion mass range $4 \cdot 10^{-15} \mathrm{eV}<m_{a}<4 \cdot 10^{-12} \mathrm{eV}$. 


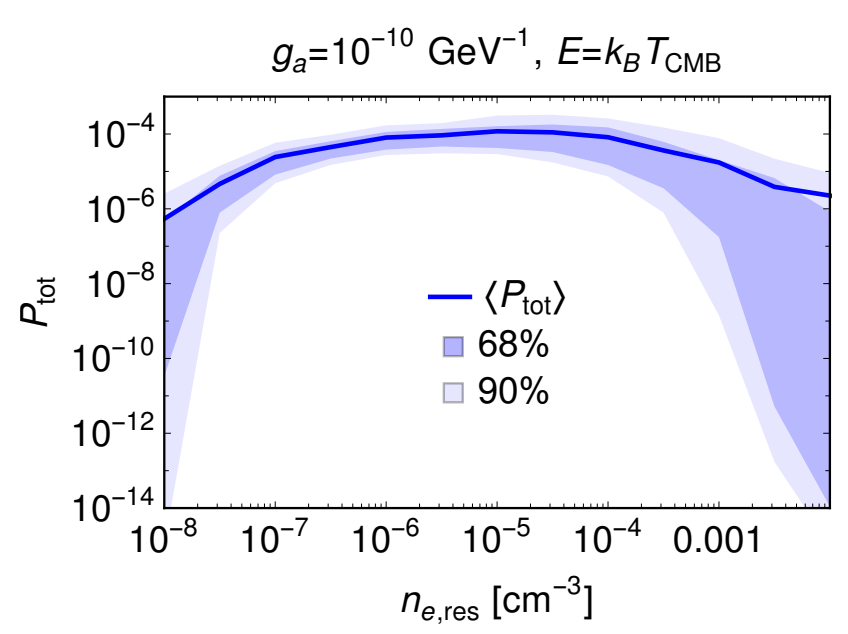

Figure 11. The conversion probability versus resonant electron number density for ALP energy $E=k_{B} T_{\mathrm{CMB}}, 1 / g_{a}=10^{10} \mathrm{GeV}$ calculated over 500 random continuous lines of sight from TNG100 up to redshift $z=6$. The blue line represents the average values, and the shaded regions correspond to $68 \%$ and $90 \%$ of conversion probabilities.

\subsection{Constraints from CMB distortions}

In this section, we consider the effect where a CMB photon converts into an axion. The probability of this conversion is proportional to the photon energy, and its occurrence induces distortions in the CMB spectrum. The strength of the effect depends on the axion coupling $g_{a}$, so we can constrain the axion model from CMB spectrum measurements obtained by COBE/FIRAS (Fixsen et al. 1996), which determined the CMB spectrum in the frequency range from 68 to $637 \mathrm{GHz}$ with a precision of up to $\Delta B_{E} / B_{E} \approx 10^{-4}$, where $B_{E}$ is a measured spectral radiance and $\Delta B_{E}$ is its uncertainty.

Resonant axion-photon conversion modifies the CMB spectrum as

$$
B_{E}(E)=B_{E}^{\mathrm{CMB}}(E)\left[1-\left\langle P_{\mathrm{tot}}(E)\right\rangle\right],
$$

where $B_{E}^{\mathrm{CMB}}$ is the spectral radiance of the initial CMB spectrum and $\left\langle P_{\text {tot }}(E)\right\rangle$ is the average conversion probability. We see that conversion produces an energy-dependent modification of the Planck spectrum. We estimate the exclusion region of the COBE/FIRAS measurement by a simple condition that follows from (7),

$$
\frac{\Delta B_{E}}{B_{E}}=\left\langle P_{\text {tot }}\left(k_{B} T_{\mathrm{CMB}}\right)\right\rangle<10^{-4},
$$

where $T_{\mathrm{CMB}}=2.7260(13) \mathrm{K}$ (Fixsen 2009).

The result for the exclusion is shown in Fig. 12, where we also add an estimation of the sensitivity of the future CMB distortion experiment PIXIE using the same condition (8) but taking an expected sensitivity for the PIXIE experiment of $\Delta B_{E} / B_{E}<$ $10^{-7}$ (Chluba et al. 2019a,b). As we can see, the CMB-based constraints are not competitive with other existing constraints. The reason for this is clear - the probability of axion-photon conversion is proportional to energy (see Eq. (6)). Therefore, much stronger constraints can be obtained from sources of photons with higher energy, e.g. X-ray or, especially, gamma-rays, see e.g. Montanino et al. (2017); Reynolds et al. (2019), where however non-resonant conversion is discussed. X-ray and gamma-ray sources are not allsky, but individual point sources, and a study of the effect of the

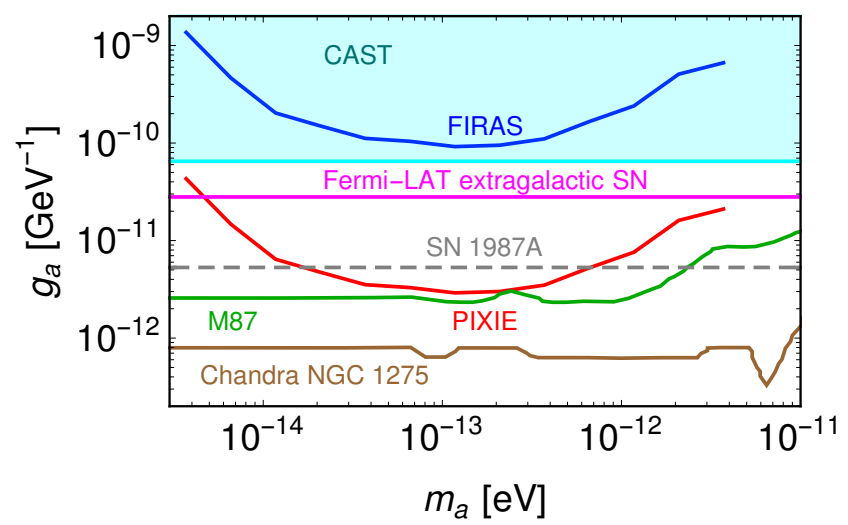

Figure 12. Constraints on the axion-photon coupling constant, $g_{a}$, as a function of axion mass, $m_{a}$, from FIRAS/COBE (blue line) and a projection of the future sensitivity of the PIXIE experiment (red line). We also present here other relevant constraints in this mass range of the axion: CAST (Anastassopoulos et al. 2017), Fermi-LAT extragalactic SN (Meyer \& Petrushevska 2020), M87 (Marsh et al. 2017), Chandra NGC 1255 (Reynolds et al. 2019).

IGM on their spectra requires a different methodology that is beyond the scope of this paper. We expect however that our results can be used for such an analysis in the future.

\section{SUMMARY, DISCUSSION AND CONCLUSIONS}

In this paper, we have quantified the effects of galaxy evolution processes on the magnetization of the intergalactic medium (IGM). To this end, we have used several simulations from the IllustrisTNG (Nelson et al. 2018; Marinacci et al. 2018) suite which all include treatment of ideal magnetohydrodynamics coupled to a state-of-the-art model for galaxy formation physics and feedback. In these calculations we always assume a homogeneous initial seed field, which is then amplified throughout the process of collapse and structure formation.

As demonstrated in previous analyses (see e.g. Pakmor et al. 2017), strong magnetic fields are produced inside galaxies due to small-scale MHD dynamos. In this paper, we have shown that such strongly amplified magnetic fields can be distributed to larger volumes due to galactic feedback, in particular feedback from supermassive black holes (see also Nelson et al. 2019b). These largescale bubbles produced by outflows from galaxies and clusters develop particularly at redshifts $z<2$, and contain magnetic fields that are several orders of magnitude stronger than in the unaffected regions of the IGM with the same electron density.

As a result, similarly to the magnetic fields inside galaxies, these fields are to a certain extent invariant with respect to the assumed initial conditions for magnetic fields (see Fig. 2 and its discussion in the text). We show that these over-magnetized bubbles with $|B| \geqslant 10^{-12} \mathrm{cG}$, enhanced metallicity, and with clear outflowing kinematic signatures, can be as large as tens of Mpc. Their existence, and extent, is directly related to feedback activity from supermassive black holes in the centers of massive galaxies. Supernovae explosions also produce similar albeit smaller magnetized bubbles around lower mass galaxies.

We study the volume filling fraction of these strong field regions and their distribution over random lines of sight. We find that a typical intergalactic line of sight at $z=0$ extends for $\sim 10-15 \%$ 
of its length within these regions for seed magnetic fields lower than $10^{-14} \mathrm{cG}$. This implies that strongly magnetized bubbles are important for the propagation of light and high-energy cosmic rays through the Universe.

We use these results from TNG to put bounds on the photonaxion conversion from spectral distortions of the CMB. The disappearance of CMB photons due to their resonant conversion into axions in the IGM introduces deviations from the black-body spectrum of the CMB. If the mass of the axion is in the range $4 \cdot 10^{-15} \mathrm{eV}<m_{a}<4 \cdot 10^{-12} \mathrm{eV}$ where many resonances happen along a typical LOS, this distortion can be above the limit of COBE/FIRAS. As the photon-axion coupling grows with energy, the bounds based on the CMB distortions are not competitive with other existing bounds obtained using much more energetic photons.

In addition to $\mathrm{CMB}$ spectrum distortion, the conversion of $\mathrm{CMB}$ photons into axions produces additional anisotropy in both the temperature and polarization of the CMB. These bounds could be stronger than those obtained using the spectrum averaged over the whole sky. Indeed, the effect of the axion-photon conversion in the IGM is dominated by the contributions of the over-magnetized bubbles discussed in the paper. On one hand, this makes this effect to some extent independent of the unknown properties of the initial seed fields and therefore justifies the use of simulations. On the other hand, this means that anisotropies are introduced on the scales that are small for the $\mathrm{CMB}$, i.e. the signal peaks at relatively high- $\ell$, where the power spectrum of CMB anisotropies is already suppressed. At the same time, the size of the bubbles is noticeably larger than that of dark matter halos themselves, so the resulting impact could be distinguished from e.g. the Sunyaev-Zeldovich effect. However, to perform such an analysis we cannot calculate the conversion probability only for infinitesimal sightlines, and must instead produce an anisotropy map for a part of the sky, which is a challenging task for future work.

Stronger constraints from photon-axion conversion in IGM could also leverage the energy dependence of the coupling constant in order to study the propagation of gamma-ray photons through the IGM (see e.g. Montanino et al. 2017 for a study of the non-resonant conversion of gamma-rays in the IGM using ENZO simulations). This involves different classes of sources and will also be studied elsewhere.

Finally, the over-magnetized bubbles may contribute to measurements of the Faraday rotation measure (RM). For example, O'Sullivan et al. (2020) studies 349 pairs of radio sources in order to separate the contribution to RM from the IGM versus from the contribution of the local environments of the source and the observer (Milky Way), aiming to put a bound on the value of the primordial seed fields. However, the contribution of the overmagnetized bubbles encountered on the way from the source to the observer may be significant and could hinder constraints on the contribution of the "true" intergalactic magnetic fields that exist in regions unaffected by outflows.

To illustrate this, we show in Fig 13 the value of the Faraday Rotation measure for 1000 LOS as a function of the distance to the source (blue shaded bands). To disentangle the contribution of the bubbles, we choose the lines of sight such that they do not have magnetic field $B>10^{-12} \mathrm{cG}$ at the beginning and at the end of the LOS. We also exclude the contribution of voxels with electron density $n_{e}>0.01 \mathrm{~cm}^{-3}$, to remove galaxies, and of voxels with magnetic fields $B<10^{-12} \mathrm{cG}$, to include only the contribution of the bubbles. We also show the region that contains $90 \%$ of values of RM of the radio sources observed by LOFAR presented in O'Sullivan et al. (2020) (red shaded band).

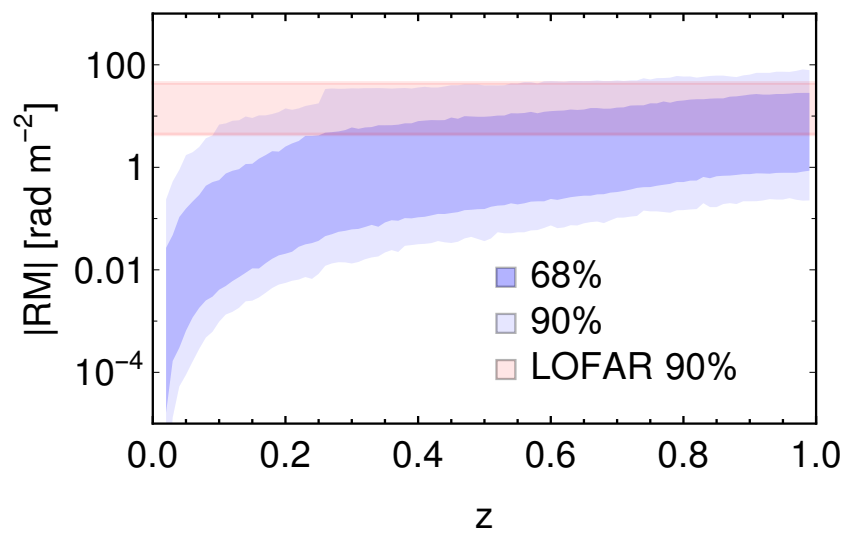

Figure 13. Distribution of the contribution to Faraday Rotation Measure from the magnetic fields in outflow-generated bubbles only (see text), for sources located at redshifts between $z=0$ and 1 calculated using 1000 random lines-of-sight from the TNG100 simulation (see 2 for description). Blue (light blue) region contains $68 \%(90 \%)$ of all values of RM that we obtained for this mock lines-of-sight. The red region contains $90 \%$ of values of RM of the observed radio sources presented in O'Sullivan et al. (2020).

We see that for the sources located at $z \geqslant 0.2$ the contribution of outflow-driven bubbles to RM measurements can be significant. This contamination must therefore be taken into account when inferring intergalactic magnetic field values from RM measurements. On the other hand, a more detailed analysis could constrain the properties (i.e. size scales, abundance) of feedback-driven bubbles themselves, thus informing models of galaxy formation and feedback, particularly when radio source counterparts with measurable distances are identifiable.

We conclude by noting that the baryonic feedback models and physics employed in the IllustrisTNG simulations are necessarily simplified treatments. In the future, more sophisticated black hole feedback models, such as those modeling unresolved accretion disks, black hole spin, and relativistic jet production could produce different emergent outflows. Similarly, the TNG galaxy formation model neglects several physical processes, most notably cosmic rays, low-temperature cooling and chemistry below $10^{4} \mathrm{~K}$, and coupled radiation-hydrodynamical interactions, which could similarly impact the generation and propagation of the outflows driven by galaxies and their supermassive black holes.

\section{ACKNOWLEDGEMENTS}

We thank Matthieu Schaller and Josef Pradler for the useful discussions. AS is supported by the FWF Research Group grant FG1. AAG, KB and AB are supported by the European Research Council (ERC) Advanced Grant "NuBSM" (694896). The TNG simulations were carried out with compute time granted by the Gauss Centre for Supercomputing (GCS) under Large-Scale Projects GCS-DWAR and GCS-ILLU on the GCS share of the supercomputer Hazel Hen at the High Performance Computing Center Stuttgart (HLRS). Additional simulations and analysis were carried out on the Isaac cluster of the Max Planck Institute for Astronomy (MPIA), and the systems of the Max Planck Computing and Data Facility (MPCDF). 


\section{DATA AVAILABILITY}

The data underlying this article is available on reasonable request.

\section{References}

Acharya B., et al., 2018, Science with the Cherenkov Telescope Array. WSP (arXiv: 1709.07997), doi:10.1142/10986

Aghanim N., et al., 2020, Astron. Astrophys., 641, A6

Alves Batista R., Shin M.-S., Devriendt J., Semikoz D., Sigl G., 2017, Phys. Rev. D, 96, 023010

Anastassopoulos V., et al., 2017, Nature Phys., 13, 584

Beck R., 2015, A\&A, 578, A93

Benitez-Llambay A., 2015, py-sphviewer: Py-SPHViewer v1.0.0, doi:10.5281/zenodo.21703, http://dx.doi.org/10.5281/ zenodo. 21703

Birk G. T., Wiechen H., Otto A., 1999, ApJ, 518, 177

Blandford R. D., Begelman M. C., 1999, MNRAS, 303, L1

Blasi P., Burles S., Olinto A. V., 1999, Astrophys. J. Lett., 514, L79

Bondarenko K., Pradler J., Sokolenko A., 2020, Phys. Lett. B, 805, 135420

Brandenburg A., Subramanian K., 2005, Phys. Rept., 417, 1

Bray J., Scaife A., 2018, Astrophys. J., 861, 3

Brentjens M. A., de Bruyn A., 2005, Astron. Astrophys., 441, 1217

Butsky I., et al., 2016, Mon. Not. Roy. Astron. Soc., 462, 663

Butsky I., Zrake J., Kim J.-h., Yang H.-I., Abel T., 2017, ApJ, 843, 113

Carilli C. L., Rawlings S., 2004, New Astron. Rev., 48, 979

Carilli C., Taylor G., 2002, Ann. Rev. Astron. Astrophys., 40, 319

Chluba J., et al., 2019a

Chluba J., et al., 2019b, Bull. Am. Astron. Soc., 51, 184

Dermer C. D., Cavadini M., Razzaque S., Finke J. D., Chiang J., Lott B., 2011, Astrophys. J. Lett., 733, L21

Dobrynina A., Kartavtsev A., Raffelt G., 2015, Phys. Rev. D, 91, 083003

Dolag K., Bartelmann M., Lesch H., 1999, A\&A, 348, 351

Dolag K., Grasso D., Springel V., Tkachev I., 2005, JCAP, 01, 009

Dolag K., Kachelriess M., Ostapchenko S., Tomas R., 2011, Astrophys. J. Lett., 727, L4

Donnari M., et al., 2020, MNRAS,

Dubois Y., Teyssier R., 2010, A\&A, 523, A72

Durrer R., Neronov A., 2013, Astron. Astrophys. Rev., 21, 62

Ferland G. J., et al., 2017, Rev. Mex. Astron. Astrofis., 53, 385

Fixsen D., 2009, Astrophys. J., 707, 916

Fixsen D., Cheng E., Gales J., Mather J. C., Shafer R., Wright E., 1996, Astrophys. J., 473, 576

Garaldi E., Pakmor R., Springel V., 2020, arXiv e-prints, p. arXiv:2010.09729

Gnedin N. Y., Ferrara A., Zweibel E. G., 2000, Astrophys. J., 539, 505

Haardt F., Madau P., 2012, ApJ, 746, 125

Hackstein S., Vazza F., Brüggen M., Sigl G., Dundovic A., 2016, Monthly Notices of the Royal Astronomical Society, 462, 3660

Heinrich C. H., Miranda V., Hu W., 2017, Phys. Rev. D, 95, 023513

Kulsrud R. M., 1999, ARA\&A, 37, 37

Kulsrud R. M., Zweibel E. G., 2008, Rept. Prog. Phys., 71, 0046091

Kulsrud R. M., Cen R., Ostriker J. P., Ryu D., 1997, Astrophys. J., 480, 481

Laing R., Bridle A., Parma P., Feretti L., Giovannini G., Murgia M., Perley R., 2008, Mon. Not. Roy. Astron. Soc., 386, 657

Marinacci F., Vogelsberger M., Mocz P., Pakmor R., 2015, Mon. Not. Roy. Astron. Soc., 453, 3999

Marinacci F., et al., 2018, MNRAS, 480, 5113

Marsh M. D., Russell H. R., Fabian A. C., McNamara B. P., Nulsen P., Reynolds C. S., 2017, JCAP, 12, 036

Martin-Alvarez S., Devriendt J., Slyz A., Teyssier R., 2018, MNRAS, 479, 3343

Martin-Alvarez S., Slyz A., Devriendt J., Gómez-Guijarro C., 2020, MNRAS, 495, 4475

Meyer M., Petrushevska T., 2020, Phys. Rev. Lett., 124, 231101

Mirizzi A., Redondo J., Sigl G., 2009, JCAP, 08, 001
Montanino D., Vazza F., Mirizzi A., Viel M., 2017, Phys. Rev. Lett., 119, 101101

Naiman J. P., et al., 2018, MNRAS, 477, 1206

Nelson D., et al., 2018, MNRAS, 475, 624

Nelson D., et al., 2019a, Computational Astrophysics and Cosmology, 6, 2

Nelson D., et al., 2019b, MNRAS, 490, 3234

Neronov A., Semikoz D., 2009, Phys. Rev. D, 80, 123012

Neronov A., Vovk I., 2010, Science, 328, 73

O’Sullivan S., et al., 2020, Mon. Not. Roy. Astron. Soc., 495, 2607

Pakmor R., Springel V., 2013, MNRAS, 432, 176

Pakmor R., Bauer A., Springel V., 2011, MNRAS, 418, 1392

Pakmor R., Marinacci F., Springel V., 2014a, Astrophys. J. Lett., 783, L20

Pakmor R., Marinacci F., Springel V., 2014b, ApJ, 783, L20

Pakmor R., et al., 2017, MNRAS, 469, 3185

Pakmor R., et al., 2020, MNRAS, 498, 3125

Parker E. N., 1955, ApJ, 122, 293

Pillepich A., et al., 2018a, MNRAS, 473, 4077

Pillepich A., et al., 2018b, MNRAS, 475, 648

Planck Collaboration Ade P. A. R., et al., 2016, A\&A, 594, A13

Preskill J., Wise M. B., Wilczek F., 1983, Phys. Lett. B, 120, 127

Pshirkov M., Tinyakov P., Urban F., 2016, Phys. Rev. Lett., 116, 191302

Raffelt G., Stodolsky L., 1988, Phys. Rev. D, 37, 1237

Ravi V., et al., 2016, Science, 354, 1249

Reynolds C. S., Marsh M. D., Russell H. R., Fabian A. C., Smith R., Tombesi F., Veilleux S., 2019, ApJ, 890

Rieder M., Teyssier R., 2016, MNRAS, 457, 1722

Rieder M., Teyssier R., 2017, MNRAS, 471, 2674

Ruzmaikin A., Sokolov D., Shukurov A., 1988, Nature, 336, 341

Sikivie P., 1983, Phys. Rev. Lett., 51, 1415

Springel V., 2010, MNRAS, 401, 791

Springel V., et al., 2018, MNRAS, 475, 676

Steinwandel U. P., Beck M. C., Arth A., Dolag K., Moster B. P., Nielaba P., 2019, MNRAS, 483, 1008

Steinwandel U. P., Dolag K., Lesch H., Moster B. P., Burkert A., Prieto A., 2020, MNRAS, 494, 4393

Su K.-Y., Hopkins P. F., Hayward C. C., Faucher-Giguère C.-A., Kereš D., Ma X., Robles V. H., 2017, MNRAS, 471, 144

Subramanian K., 2016, Rept. Prog. Phys., 79, 076901

Subramanian K., Narasimha D., Chitre S., 1994, Monthly Notices of the Royal Astronomical Society, 271, L15

Tavecchio F., Ghisellini G., Bonnoli G., Foschini L., 2011, Mon. Not. Roy. Astron. Soc., 414, 3566

Taylor A., Vovk I., Neronov A., 2011, Astron. Astrophys., 529, A144

Vazza F., Brüggen M., Gheller C., Hackstein S., Wittor D., Hinz P., 2017, Class. Quant. Grav., 34, 234001

Vazza F., Brüggen M., Hinz P. M., Wittor D., Locatelli N., Gheller C., 2018, MNRAS, 480, 3907

Vernstrom T., Gaensler B., Rudnick L., Andernach H., 2019, Astrophys. J., 878,92

Weinberg S., 1978, Phys. Rev. Lett., 40, 223

Weinberger R., et al., 2017, MNRAS, 465, 3291

Weinberger R., et al., 2018, MNRAS, 479, 4056

Wilczek F., 1978, Phys. Rev. Lett., 40, 279

Yuan F., Narayan R., 2014, ARA\&A, 52, 529

van Weeren R., de Gasperin F., Akamatsu H., Brüggen M., Feretti L., Kang H., Stroe A., Zandanel F., 2019, Space Sci. Rev., 215, 16

\section{APPENDIX A: VARYING THE INITIAL SEED FIELD WITH 25 CMPC/H SIMULATIONS}

An important aspect of our work is to determine dependencies on the initial conditions of the magnetic field seed. For this, we use the TNG model variations for four different values of initial seeds. Each contains $512^{3}$ gas and dark matter particles, within a box of $L \sim 25 \mathrm{cMpc} / \mathrm{h}$, making the resolution similar to that of TNG100. 

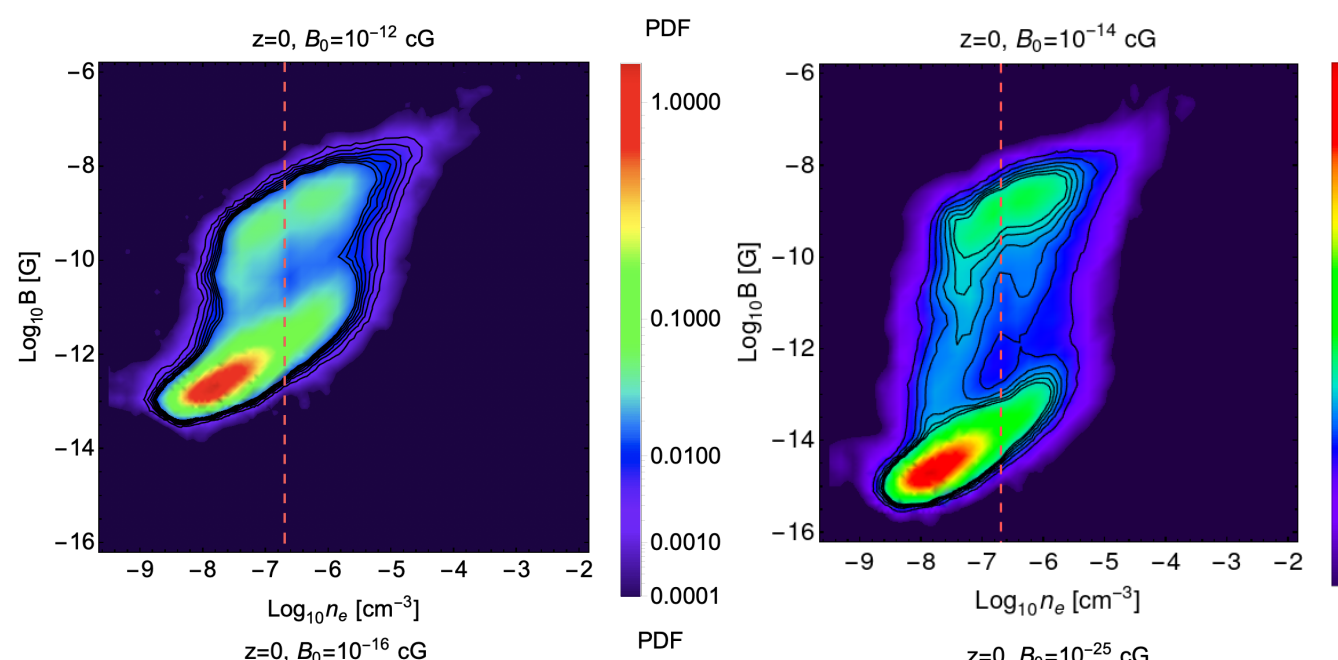

PDF
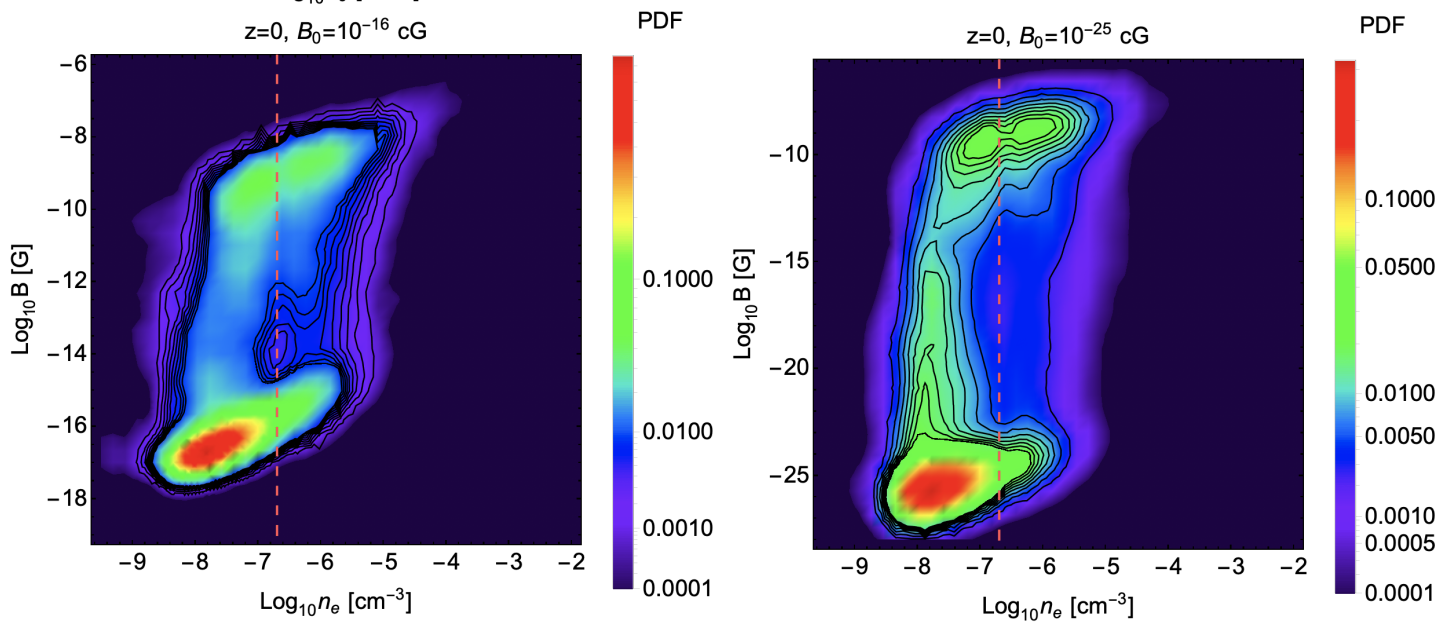

Figure A1. Distribution of the magnetic field magnitude and electron number density in the fiducial $25 \mathrm{cMpc} / \mathrm{h}$ simulation at redshift $z=0$ for different values of seed field: $10^{-12} \mathrm{cG}, 10^{-14} \mathrm{cG}, 10^{-16} \mathrm{cG}$, and $10^{-25} \mathrm{cG}$. The red dashed line represents the average electron number density.

In Fig. A1 we present for these simulations the analogous result as in Fig. 3 for $z=0$. The initial conditions of the magnetic field seed appear to affect mainly the lower branch while the upper branch occupies magnetic field values around $10^{-9} \mathrm{G}$ which is in concordance with our result presented in Fig. 2. In Fig. A2 we show the volume fractions for each variation as seen in Fig. 4. We see that the volume filling fraction weakly depends on the seed magnetic field value, and the results for the $25 \mathrm{cMpc} / \mathrm{h}$ volume are in good agreement with the fiducial TNG100 simulation adopted throughout.

\section{APPENDIX B: EVOLUTION OF MAGNETIC FIELD STRENGTH WITH REDSHIFT}

We present in Fig. B1 the time progression for the correlation of electron number density and the magnitude of the magnetic field for the TNG100 simulation. We can observe how the upper branch develops with time as the universe evolves and becomes clearly distinguishable for $z \lesssim 1$. Similarly, Fig. B2 shows the evolution of volume filling fraction for large values of magnetic fields.

Fig. B3 displays the evolution of the length fraction of the LOS that has a magnitude of a magnetic field larger than $10^{-12} \mathrm{cG}$. We see that the distribution starts to broaden circa $z \approx 2$.

\section{APPENDIX C: TNG300: DEPENDENCE OF THE MAGNETIC FIELD ON BOX SIZE}

To explore the impact of the finite simulation size, we repeat our analysis on the larger TNG300 simulation, which however is performed at somewhat lower mass resolution than the TNG100 box: namely, dark matter and baryonic mass resolution of $5.9 \times 10^{7} \mathrm{M}_{\odot}$ and $1.1 \times 10^{7} \mathrm{M}_{\odot}$ respectively. In Fig. $\mathrm{C} 1$ we present the magnitude of the magnetic field versus the electron number density. Comparing with the results for the same redshift shown in Fig.3, we observe the same bimodal distribution within the same value ranges for both the TNG100 and TNG300-1 simulations. Comparing Fig. C2 (left) and Fig. 4 we see that TNG300 has smaller volume filling fraction (for example, for $B>10^{-12}$ the volume filling fraction in TNG100 is 0.14, while in TNG300 is 0.135 ). We believe that this is an effect of poorer resolution of the TNG300 simulation in comparison to the fiducial TNG100. 

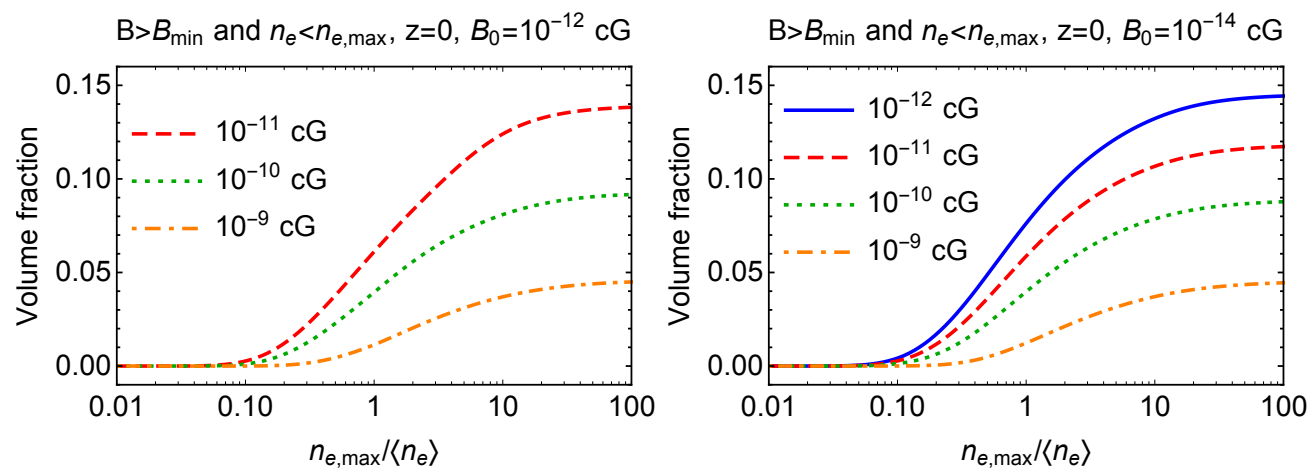

$\mathrm{B}>B_{\min }$ and $n_{e}<n_{e, \max }, \mathrm{z}=0, B_{0}=10^{-16} \mathrm{cG}$

$\mathrm{B}>B_{\text {min }}$ and $n_{e}<n_{e, \max }, \mathrm{z}=0, B_{0}=10^{-25} \mathrm{cG}$
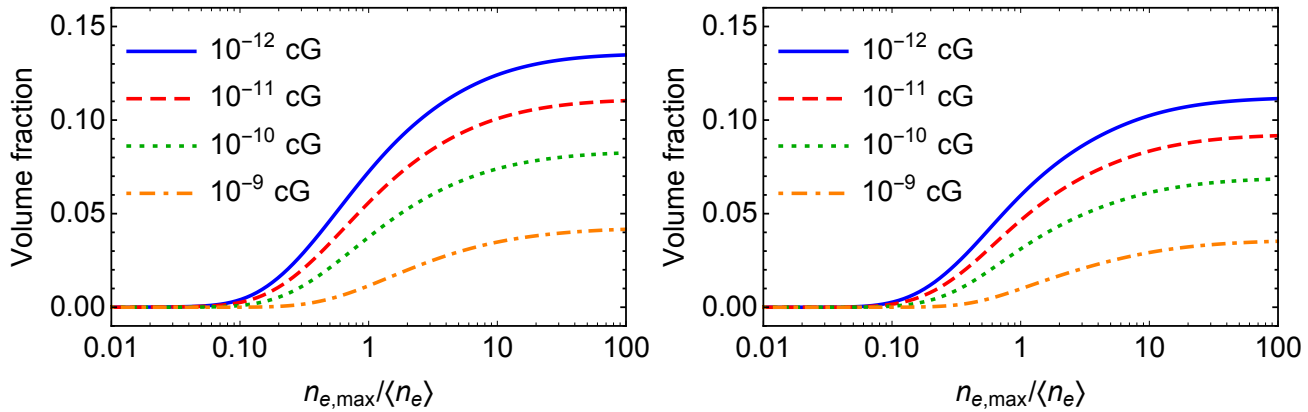

Figure A2. Volume fractions of the regions where the magnetic field is larger than $B_{\min }$ (legend) and the electron number density is smaller than $n_{e, \max }$ (x-axis) for $z=0$ for different values of seed field: $10^{-12} \mathrm{cG}, 10^{-14} \mathrm{cG}, 10^{-16} \mathrm{cG}$, and $10^{-25} \mathrm{cG}$. To produce these figures we used all gas cells in each of the simulation volumes. 


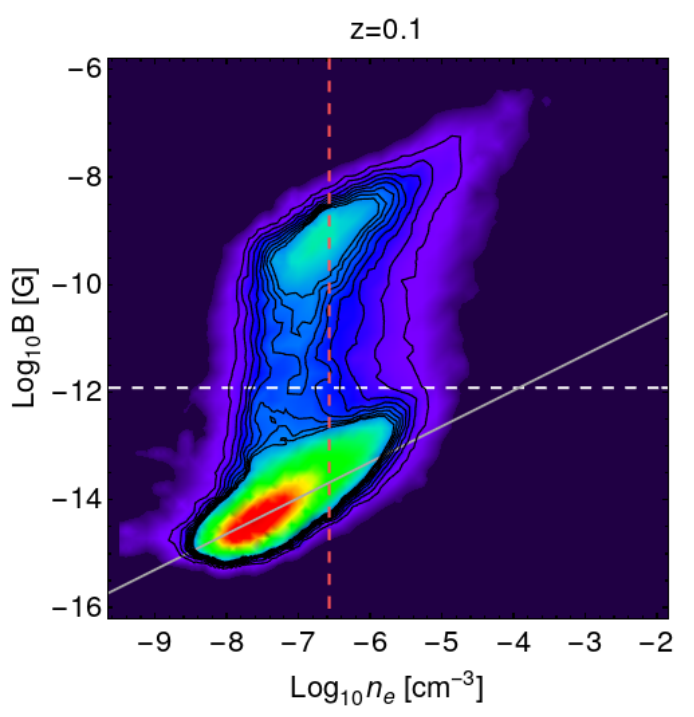

PDF
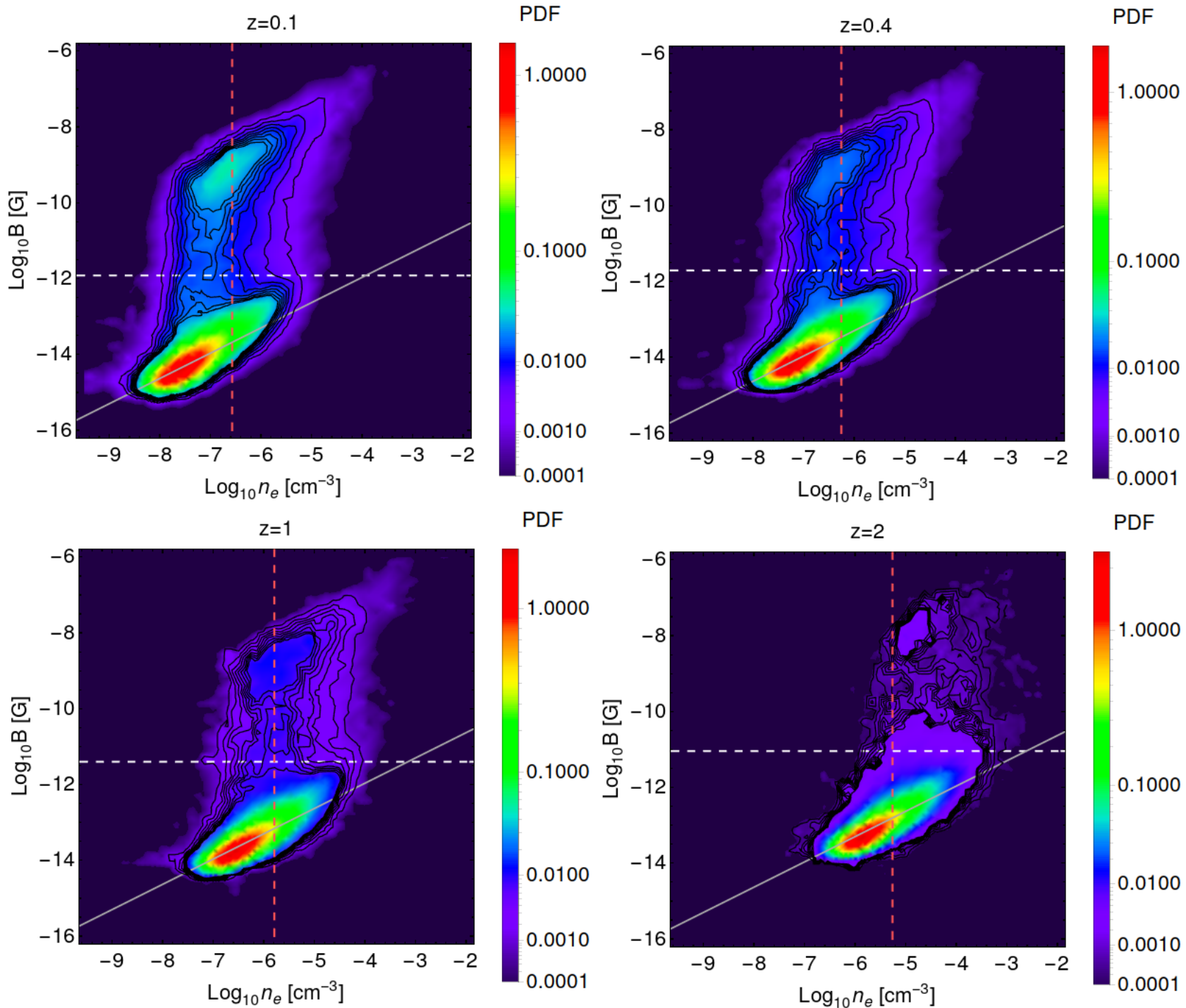

PDF
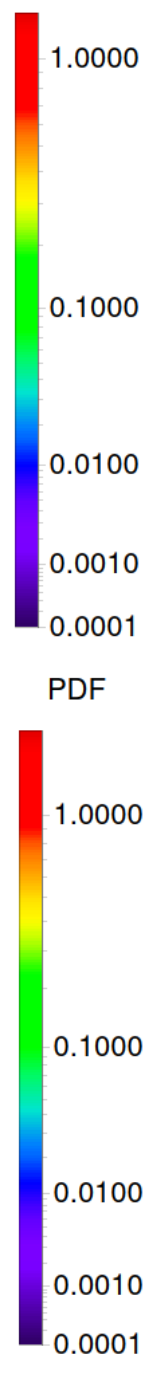

$z=2$

PDF

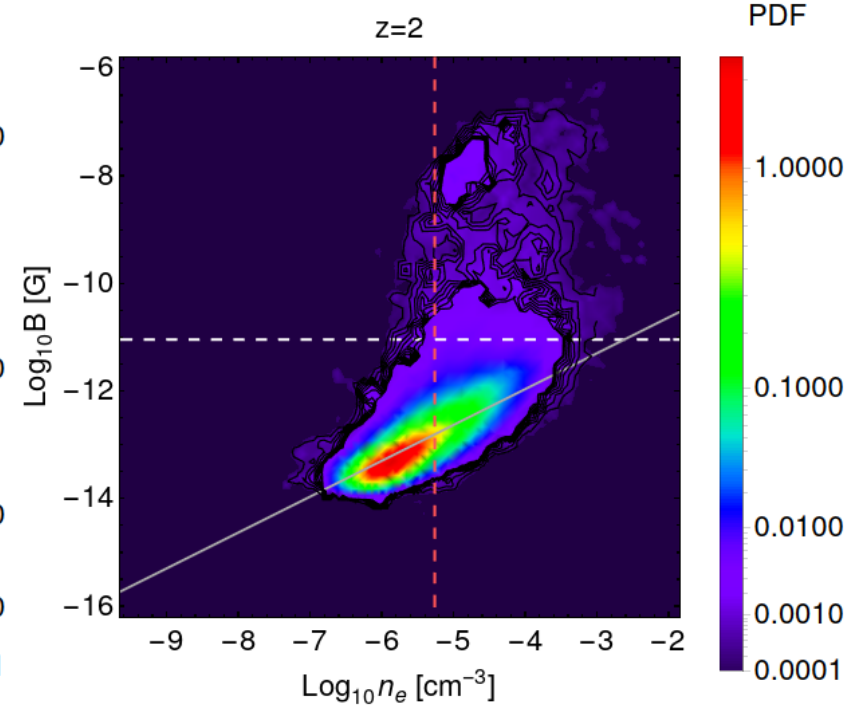

$z=3$

PDF
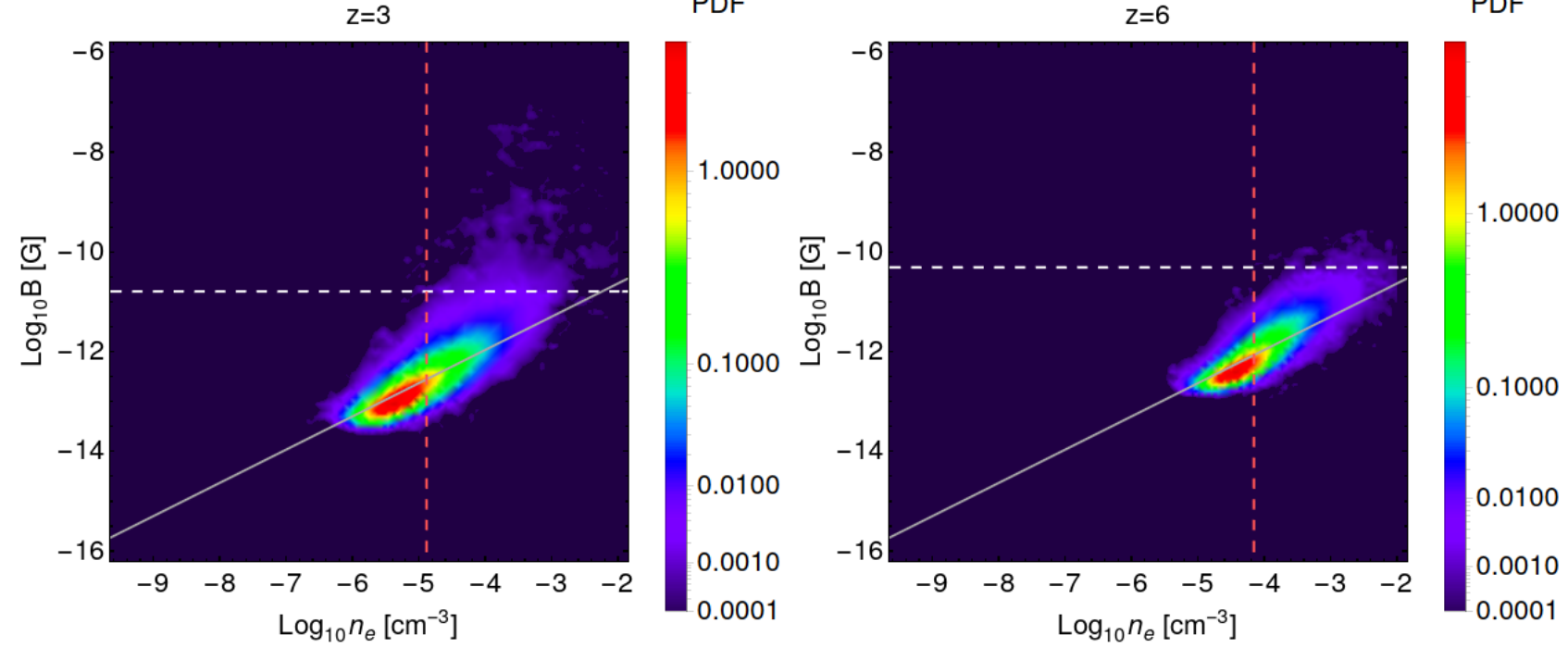

Figure B1. Distribution of the magnetic field magnitude and electron number density in the TNG100 simulation using data along 200 random lines of sight in the box at redshifts $z=0.1,0.4,1,2,3$, and 6 . The white dashed line corresponds to the comoving magnetic field value $10^{-12}$ cG that we use as smallest values of the outflow-generated magnetic field in this work. The red dashed line represents the average electron number density at a given redshift. The gray dashed line show a power law $B \propto n_{e}^{2 / 3}$, that should work for the adiabatic evolution. The seed field is $B_{0}=10^{-14} \mathrm{cG}$. 

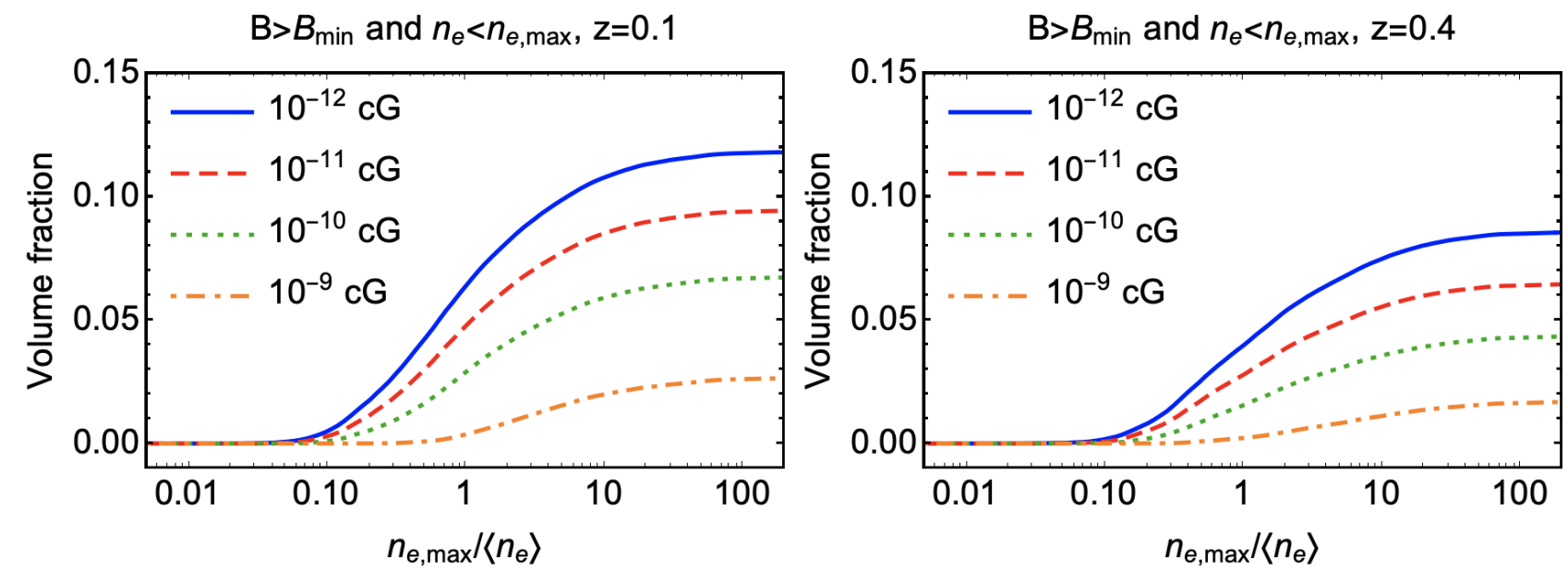

$\mathrm{B}>B_{\min }$ and $n_{e}<n_{e, \max }, \mathrm{z}=1$

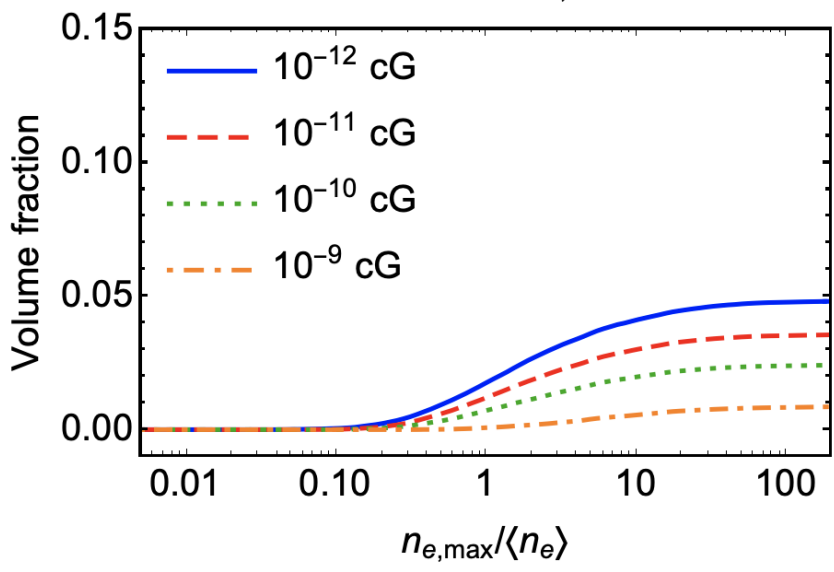

$\mathrm{B}>B_{\min }$ and $n_{e}<n_{e, \max }, \mathrm{z}=2$

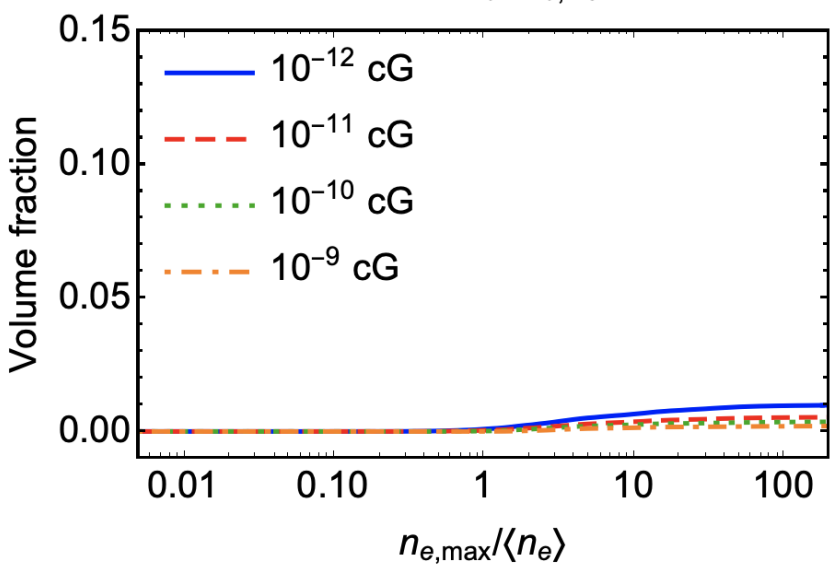

$\mathrm{B}>B_{\min }$ and $n_{e}<n_{e, \max }, \mathrm{z}=3$
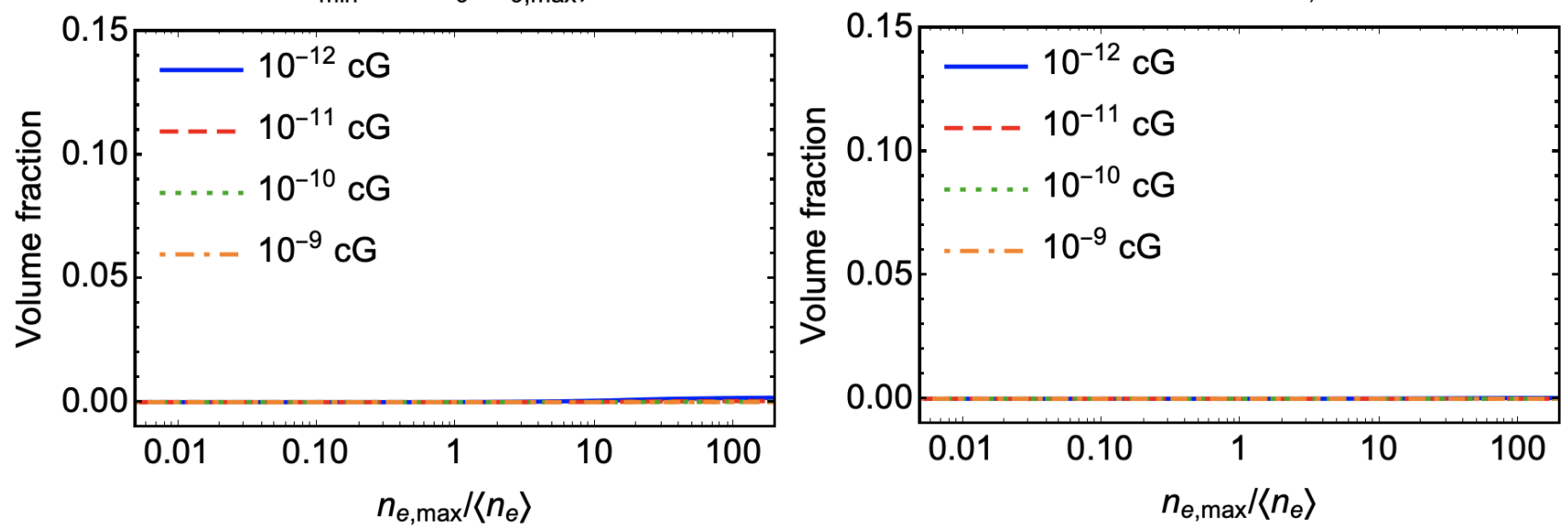

Figure B2. Volume fractions of the regions where magnetic field is larger than $B_{\min }$ and electron number density is smaller than $n_{e, \text { max }}$ as a function of redshift (different panels), from $z=0.1$ to $z=6$ using data along 200 random lines of sight in the TNG100 box. The seed field is $B_{0}=10^{-14} \mathrm{cG}$. 

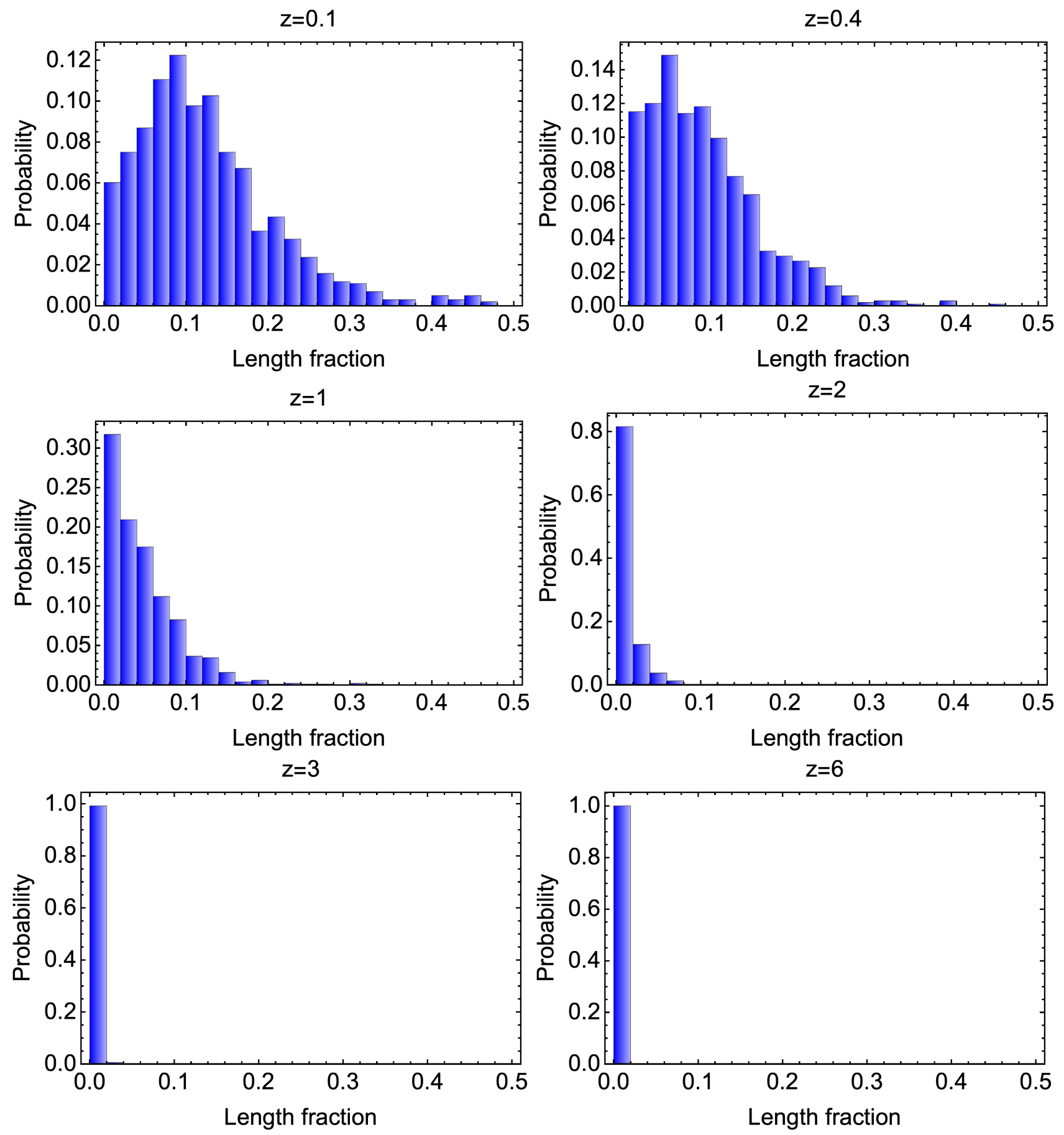

Figure B3. Probability to find a fractional length along the line of sight with magnetic field larger than $10^{-12}$ comoving Gauss for the 100 Mpc box at redshifts from $z=0.1$ to $z=6$. At each figure 1000 random lines of sight were taken from the simulation. 


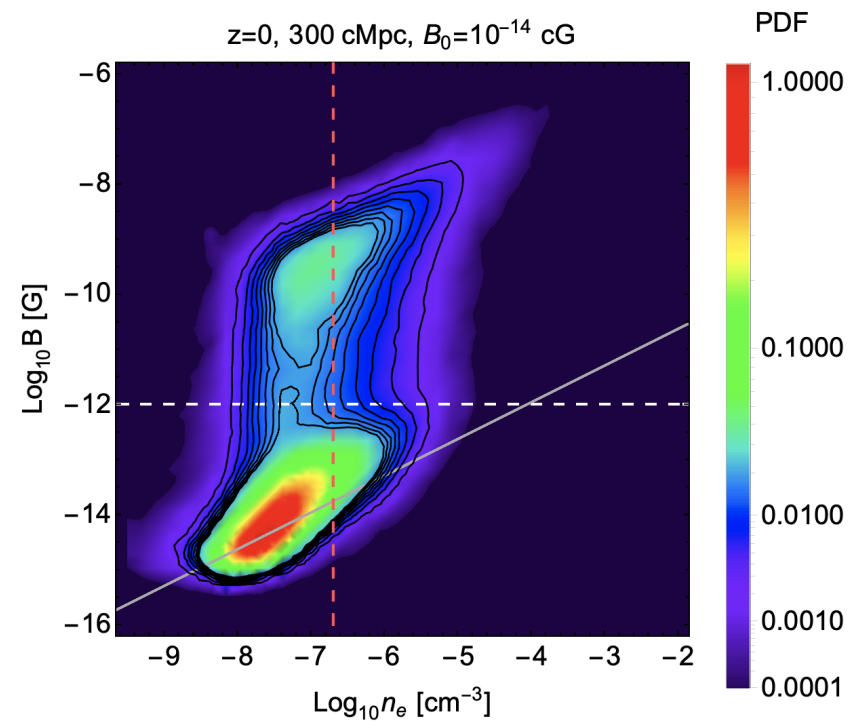

Figure C1. Distribution of the magnetic field strength and electron number density in the TNG300 simulation using data along 420 random lines of sight in the simulation box at $z=0$, with the seed field of $B_{0}=10^{-14} \mathrm{cG}$. The dashed white line corresponds to the comoving magnetic field value $10^{-12} \mathrm{cG}$ that we use as the smallest value of outflow-generated magnetic fields in this work. The red dashed line represents the average electron number density at a given redshift. The gray dashed line show a power law $B \propto n_{e}^{2 / 3}$ for the adiabatic evolution. 

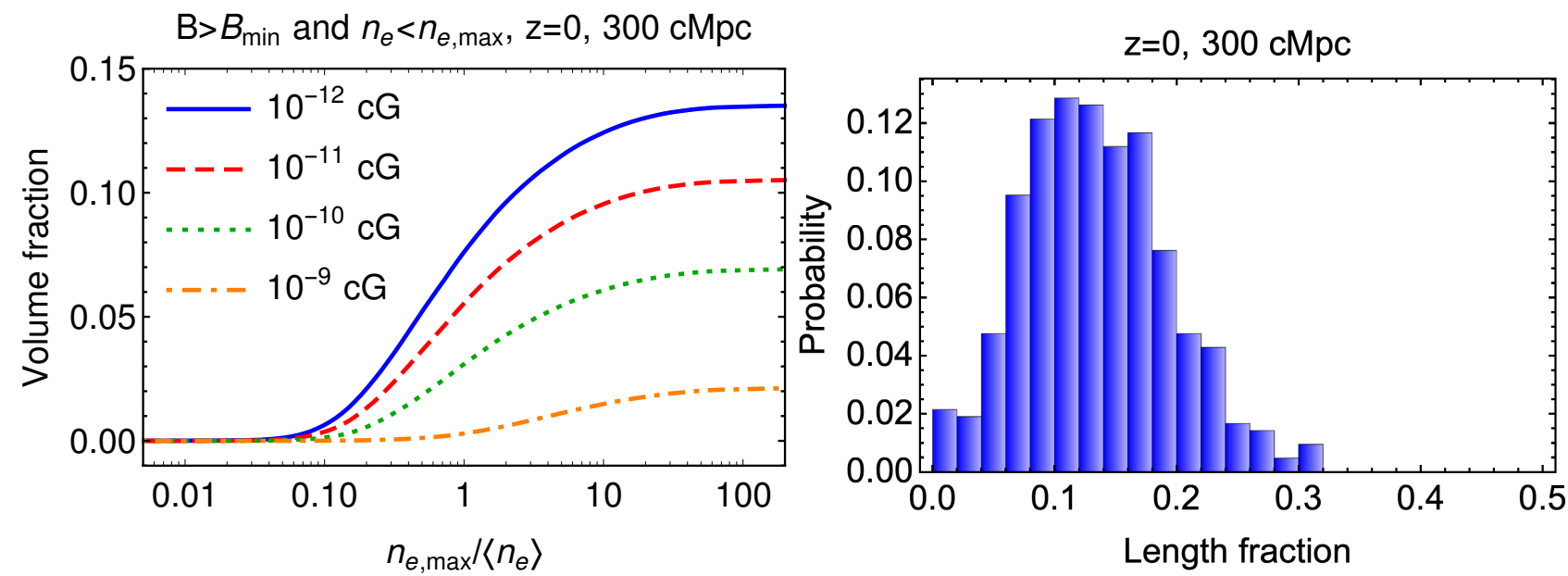

Figure C2. Left panel: volume fractions of the regions where magnetic field is larger than $B_{\min }$ and electron number density is smaller than $n_{e, \text { max }}$ for $z=0$ in the $300 \mathrm{cMpc}$ box. The seed field is $B_{0}=10^{-14} \mathrm{cG}$, as in TNG100. Right panel: Probability to find a given fractional length along the line of sight with magnetic field larger than $10^{-12}$ comoving Gauss for the $300 \mathrm{Mpc}$ box at redshift $z=0$, based on 420 random lines of sight. 